

\title{
Quiver Grassmannians and degenerate flag varieties
}

\author{
Giovanni Cerulli Irelli, Evgeny Feigin and Markus Reineke
}

\begin{abstract}
Quiver Grassmannians are varieties parametrizing subrepresentations of a quiver representation. It is observed that certain quiver Grassmannians for type A quivers are isomorphic to the degenerate flag varieties investigated earlier by Feigin. This leads to the consideration of a class of Grassmannians of subrepresentations of the direct sum of a projective and an injective representation of a Dynkin quiver. It is proved that these are (typically singular) irreducible normal local complete intersection varieties, which admit a group action with finitely many orbits and a cellular decomposition. For type A quivers, explicit formulas for the Euler characteristic (the median Genocchi numbers) and the Poincaré polynomials are derived.
\end{abstract}

\section{Introduction}

Motivation. Quiver Grassmannians, which are varieties parametrizing subrepresentations of a quiver representation, first appeared in [Crawley-Boevey 1989; Schofield 1992] in relation to questions on generic properties of quiver representations. It was observed in [Caldero and Chapoton 2006] that these varieties play an important role in cluster algebra theory [Fomin and Zelevinsky 2002]; namely, the cluster variables can be described in terms of the Euler characteristic of quiver Grassmannians. Subsequently, specific classes of quiver Grassmannians (for example, varieties of subrepresentations of exceptional quiver representations) were studied by several authors, with the principal aim of computing their Euler characteristic explicitly; see for example [Caldero and Reineke 2008; Cerulli Irelli 2011; Cerulli Irelli and Esposito 2010; Cerulli Irelli et al. 2010]. In recent papers, authors noticed that also the Poincaré polynomials of quiver Grassmannians play an important role in the study of quantum cluster algebras [Qin 2010; Berenstein and Zelevinsky 2005].

This paper originated with the observation that certain quiver Grassmannians can be identified with the $\mathfrak{s l}_{n}$-degenerate flag variety of [Feigin 2010; 2011; Feigin

The work of Evgeny Feigin was partially supported by the RFBR Grant 09-01-00058, by the grant Scientific Schools 6501.2010.2, and by the Dynasty Foundation.

MSC2010: primary 14M15; secondary 16G20.

Keywords: flag variety, quiver grassmannian, degeneration. 
and Finkelberg 2011]. This led to the consideration of a class of Grassmannians of subrepresentations of the direct sum of a projective and an injective representation of a Dynkin quiver. It turns out that this class of varieties enjoys many of the favorable properties of quiver Grassmannians for exceptional representations. More precisely, they turn out to be (typically singular) irreducible normal local complete intersection varieties which admit a group action with finitely many orbits and a cellular decomposition. The proofs of the basic geometric properties are based on generalizations of the techniques of [Reineke 2008], where the case of Grassmannians of subrepresentations of injective quiver representations is treated.

Main results. Let $Q$ be a quiver with set of vertices $Q_{0}$ of cardinality $n$ and finite set of arrows $Q_{1}$. For a representation $M$ of $Q$, we denote by $M_{i}$ the space in $M$ attached to the $i$-th vertex, and by $M_{\alpha}: M_{i} \rightarrow M_{j}$ the linear map attached to an arrow $\alpha: i \rightarrow j$. We also denote by $\left\langle_{-},,_{-}\right\rangle$the Euler form on $\mathbb{Z} Q_{0}$. Given a dimension vector $\boldsymbol{e}=\left(e_{1}, \ldots, e_{n}\right) \in \mathbb{Z}_{\geq 0} Q_{0}$ and a representation $M$ of $Q$, the quiver Grassmannian $\mathrm{Gr}_{\boldsymbol{e}}(M) \subset \prod_{i=1}^{n} \mathrm{Gr}_{e_{i}}\left(M_{i}\right)$ is the subvariety of collections of subspaces $V_{i} \subset M_{i}$ subject to the conditions $M_{\alpha} V_{i} \subset V_{j}$ for all $\alpha: i \rightarrow j \in Q_{1}$. In this paper we study a certain class of quiver Grassmannians for Dynkin quivers $Q$. Before describing this class, we first consider the following example.

Let $Q$ be an equioriented quiver of type $A_{n}$ with vertices $i=1, \ldots, n$ and arrows $i \rightarrow i+1$, and let $\mathbb{C} Q$ be the path algebra of $Q$. Then the quiver Grassmannian $\mathrm{Gr}_{\operatorname{dim} \mathbb{C} Q}\left(\mathbb{C} Q \oplus \mathbb{C} Q^{*}\right)$ is isomorphic to the complete degenerate flag variety $\mathcal{F}_{n+1}^{a}$ for $G=\mathrm{SL}_{n+1}$. We recall the definition from [Feigin 2010; 2011]. Let $W$ be an $(n+1)$-dimensional vector space with basis $w_{1}, \ldots, w_{n+1}$. Let $\operatorname{pr}_{k}: W \rightarrow W$ for $k=1, \ldots, n+1$ be the projection operators $\operatorname{pr}_{k}\left(\sum_{i=1}^{n+1} c_{i} w_{i}\right)=a \sum_{i \neq k} c_{i} w_{i}$. Then the degenerate flag variety consists of collections $\left(V_{1}, \ldots, V_{n}\right)$ with $V_{i} \subset W$ and $\operatorname{dim} V_{i}=i$, subject to the conditions $\operatorname{pr}_{k+1} V_{k} \subset V_{k+1}$ for $k=1, \ldots, n-1$. These varieties are irreducible singular algebraic varieties enjoying many nice properties. In particular, they are flat degenerations of classical flag varieties $\mathrm{SL}_{n+1} / B$. Now consider the representation $M$ of $Q$ such that $M_{i}=W$ and the maps $M_{i} \rightarrow M_{i+1}$ are given by $\mathrm{pr}_{i+1}$. For example, for $n=3, M$ has the following coefficient quiver:

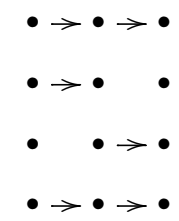

where each dot represents basis vectors $w_{1}, w_{2}, w_{3}, w_{4}$ from bottom to top and arrows represent maps. Note that $M$ is isomorphic to $\mathbb{C} Q \oplus \mathbb{C} Q^{*}$ as a representation 
of $Q$ and moreover, we have

$$
\mathcal{F}_{n+1}^{a} \simeq \mathrm{Gr}_{\operatorname{dim} \mathbb{C} Q}\left(\mathbb{C} Q \oplus \mathbb{C} Q^{*}\right) .
$$

Now let $Q$ be a Dynkin quiver. Recall that the path algebra $\mathbb{C} Q$ (resp. its linear dual $\mathbb{C} Q^{*}$ ) is isomorphic as a representation of $Q$ to the direct sum of all indecomposable projective (resp. injective) representations. Motivated by the isomorphism (1-1), we consider the quiver Grassmannians $\mathrm{Gr}_{\operatorname{dim} P}(P \oplus I)$, where $P$ and $I$ are projective resp. injective representations of $Q$. (We note that some of our results are valid for more general Grassmannians and we discuss it in the main body of the paper. However, in the introduction we restrict ourselves to the above mentioned class of varieties). We use the isomorphism (1-1) in two different ways. On the one hand, we generalize and expand the results about $\mathcal{F}_{n+1}^{a}$ to the case of the above quiver Grassmannians. On the other hand, we use general results and constructions from the theory of quiver representations to understand better the structure of $\mathcal{F}_{n+1}^{a}$.

Our first theorem is as follows:

Theorem 1.1. The variety $\operatorname{Gr}_{\operatorname{dim} P}(P \oplus I)$ is of dimension $\langle\operatorname{dim} P, \operatorname{dim} I\rangle$ and irreducible. It is a normal local complete intersection variety.

Our next goal is to construct cellular decompositions of the quiver Grassmannians and to compute their Poincaré polynomials. Let us consider the following stratification of $\operatorname{Gr}_{\operatorname{dim} P}(P \oplus I)$. For a point $N$ we set $N_{I}=N \cap I, N_{P}=\pi N$, where $\pi: P \oplus I \rightarrow P$ is the projection. Then for a dimension vector $\boldsymbol{f} \in \mathbb{Z}_{\geq 0} Q_{0}$ we set

$$
\mathscr{Y}_{\boldsymbol{f}}=\left\{N \in \mathrm{Gr}_{\operatorname{dim} P}(P \oplus I): \operatorname{dim} N_{I}=\boldsymbol{f}, \operatorname{dim} N_{P}=\operatorname{dim} P-\boldsymbol{f}\right\} .
$$

We have natural surjective maps $\zeta_{f}: \mathscr{Y}_{f} \rightarrow \operatorname{Gr}_{f}(I) \times \operatorname{Gr}_{\operatorname{dim} P-f}(P)$.

Theorem 1.2. The map $\zeta_{f}$ is a vector bundle. The fiber over a point $\left(N_{P}, N_{I}\right)$ is isomorphic to $\operatorname{Hom}_{Q}\left(N_{P}, I / N_{I}\right)$ which has dimension $\langle\operatorname{dim} P-\boldsymbol{f}, \operatorname{dim} I-\boldsymbol{f}\rangle$.

Using this theorem, we construct a cellular decomposition for each stratum $X_{f}$ and thus for the whole variety $X$ as well. Moreover, since the Poincare polynomials of $\mathrm{Gr}_{f}(I)$ and of $\mathrm{Gr}_{\operatorname{dim} P-f}(P)$ can be easily computed, we arrive at a formula for the Poincare polynomial (and thus for the Euler characteristic) of $X$. Recall (see [Feigin 2010]) that the Euler characteristic of the variety $\mathcal{F}_{n+1}^{a}$ is given by the normalized median Genocchi number $h_{n+1}$ (see [Dellac 1900; Dumont 1974; Dumont and Randrianarivony 1994; Dumont and Zeng 1994; Viennot 1982]). Using Theorem 1.2 we obtain an explicit formula for $h_{n+1}$ in terms of binomial coefficients. Moreover, we give a formula for the Poincaré polynomial of $\mathcal{F}_{n+1}^{a}$, providing a natural $q$-version of $h_{n+1}$. 
Finally we study the action of the group of automorphisms $\operatorname{Aut}(P \oplus I)$ on the quiver Grassmannians. Let $G \subset \operatorname{Aut}(P \oplus I)$ be the group

$$
G=\left[\begin{array}{cc}
\operatorname{Aut}_{Q}(P) & 0 \\
\operatorname{Hom}_{Q}(P, I) & \operatorname{Aut}_{Q}(I)
\end{array}\right] .
$$

(We note that $G$ coincides with the whole group of automorphisms unless $Q$ is of type $A_{n}$.) We prove the following theorem:

Theorem 1.3. The group $G$ acts on $\operatorname{Gr}_{\operatorname{dim} P}(P \oplus I)$ with finitely many orbits, parametrized by pairs of isomorphism classes $\left(\left[Q_{P}\right],\left[N_{I}\right]\right)$ such that $Q_{P}$ is a quotient of $P, N_{I}$ is a subrepresentation of $I$, and $\operatorname{dim} Q_{P}=\operatorname{dim} N_{I}$. Moreover, if $Q$ is equioriented of type $A_{n}$, then the orbits are cells parametrized by torus fixed points.

Outline of the paper. In Section 2, we recall general facts about quiver Grassmannians and degenerate flag varieties. In Section 3, we prove that the quiver Grassmannians $\operatorname{Gr}_{\operatorname{dim}}(P \oplus I)$ are locally complete intersections and that they are flat degenerations of the Grassmannians in exceptional representations. In Section 4, we study the action of the automorphism group on $\operatorname{Gr}_{\operatorname{dim}}(P \oplus I)$, describe the orbits and prove the normality of $\operatorname{Gr}_{\operatorname{dim}}(P \oplus I)$. In Section 5, we construct a one-dimensional torus action on our quiver Grassmannians such that the attracting sets form a cellular decomposition. Sections 6 and 7 are devoted to the case of the equioriented quiver of type $A$. In Section 6, we compute the Poincaré polynomials of $\operatorname{Gr}_{\operatorname{dim}}(P \oplus I)$ and derive several new formulas for the Euler characteristics the normalized median Genocchi numbers. In Section 7 we prove that the orbits studied in Section 4, are cells coinciding with the attracting cells constructed in Section 5. We also describe the connection with the degenerate group $\mathrm{SL}_{n+1}^{a}$.

\section{General facts on quiver Grassmannians and degenerate flag varieties}

General facts on quivers. Let $Q$ be a finite quiver with a finite set of vertices $Q_{0}$ and finite set of arrows $Q_{1}$; arrows will be written as $(\alpha: i \rightarrow j) \in Q_{1}$ for $i, j \in Q_{0}$. We assume $Q$ to be without oriented cycles. Denote by $\mathbb{Z} Q_{0}$ the free abelian group generated by $Q_{0}$, and by $\mathbb{N} Q_{0}$ the subsemigroup of dimension vectors $\boldsymbol{d}=\left(d_{i}\right)_{i \in Q_{0}}$ for $Q$. Let $\left\langle_{-},{ }_{-}\right\rangle$be the Euler form on $\mathbb{Z} Q_{0}$ defined by

$$
\langle\boldsymbol{d}, \boldsymbol{e}\rangle=\sum_{i \in Q_{0}} d_{i} e_{i}-\sum_{(\alpha: i \rightarrow j) \in Q_{1}} d_{i} e_{j}
$$

We consider finite dimensional representations $M$ of $Q$ over the complex numbers, viewed either as finite dimensional left modules over the path algebra $\mathbb{C} Q$ of $Q$, or as tuples $M=\left(\left(M_{i}\right)_{i \in Q_{0}},\left(M_{\alpha}: M_{i} \rightarrow M_{j}\right)_{(\alpha: i \rightarrow j) \in Q_{1}}\right)$ consisting of finite dimensional complex vector spaces $M_{i}$ and linear maps $M_{\alpha}$. The category $\operatorname{rep}(Q)$ of all such 
representations is hereditary (that is, $\operatorname{Ext}_{\bar{Q}}^{\geq 2}\left({ }_{-},{ }_{-}\right)=0$ ). Its Grothendieck group $K(\operatorname{rep}(Q))$ is isomorphic to $\mathbb{Z} Q_{0}$ through the the identification of the class of a representation $M$ with its dimension vector $\operatorname{dim} M=\left(\operatorname{dim} M_{i}\right)_{i \in Q_{0}} \in \mathbb{Z} Q_{0}$. The Euler form defined above then identifies with the homological Euler form, that is,

$$
\operatorname{dim} \operatorname{Hom}_{Q}(M, N)-\operatorname{dim}_{E^{2}}^{1}(M, N)=\langle\operatorname{dim} M, \operatorname{dim} N\rangle
$$

for all representations $M$ and $N$.

Associated to a vertex $i \in Q_{0}$, we have the simple representation $S_{i}$ of $Q$ with $\left(\operatorname{dim} S_{i}\right)_{j}=\delta_{i, j}$ (the Kronecker delta), the projective indecomposable $P_{i}$, and the indecomposable injective $I_{i}$. The latter are determined as the projective cover (resp. injective envelope) of $S_{i}$; more explicitly, $\left(P_{i}\right)_{j}$ is the space generated by all paths from $i$ to $j$, and the linear dual of $\left(I_{i}\right)_{j}$ is the space generated by all paths from $j$ to $i$.

Given a dimension vector $\boldsymbol{d} \in \mathbb{N} Q_{0}$, we fix complex vector spaces $M_{i}$ of dimension $d_{i}$ for all $i \in Q_{0}$. We consider the affine space

$$
R_{\boldsymbol{d}}(Q)=\bigoplus_{(\alpha: i \rightarrow j)} \operatorname{Hom}_{\mathbb{C}}\left(M_{i}, M_{j}\right)
$$

its points canonically parametrize representations of $Q$ of dimension vector $\boldsymbol{d}$. The reductive algebraic group $G_{\boldsymbol{d}}=\prod_{i \in Q_{0}} \mathrm{GL}\left(M_{i}\right)$ acts naturally on $R_{\boldsymbol{d}}(Q)$ via base change

$$
\left(g_{i}\right)_{i} \cdot\left(M_{\alpha}\right)_{\alpha}=\left(g_{j} M_{\alpha} g_{i}^{-1}\right)_{(\alpha: i \rightarrow j)},
$$

such that the orbits $\mathrm{O}_{M}$ for this action naturally correspond to the isomorphism classes $[M]$ of representations of $Q$ of dimension vector $\boldsymbol{d}$. Note that $\operatorname{dim} G_{\boldsymbol{d}}-$ $\operatorname{dim} R_{\boldsymbol{d}}(Q)=\langle\boldsymbol{d}, \boldsymbol{d}\rangle$. The stabilizer under $G_{\boldsymbol{d}}$ of a point $M \in R_{\boldsymbol{d}}(Q)$ is isomorphic to the automorphism group $\operatorname{Aut}_{Q}(M)$ of the corresponding representation, which (being open in the endomorphism space $\operatorname{End}_{Q}(M)$ ) is a connected algebraic group of dimension $\operatorname{dim} \operatorname{End}_{Q}(M)$. In particular, we get the following formulas:

$$
\operatorname{dim} \mathfrak{O}_{M}=\operatorname{dim} G_{d}-\operatorname{dim} \operatorname{End}_{Q}(M), \operatorname{codim}_{R_{d}} \mathcal{O}_{M}=\operatorname{dim}_{\operatorname{Ext}_{Q}}^{1}(M, M) .
$$

Basic facts on quiver Grassmannians. The constructions and results in this section follow [Caldero and Reineke 2008; Schofield 1992]. Additionally to fix another dimension vector $\boldsymbol{e}$ such that $\boldsymbol{e} \leq \boldsymbol{d}$ componentwise, and define the $Q_{0}$-graded Grassmannian $\operatorname{Gr}_{\boldsymbol{e}}(\boldsymbol{d})=\prod_{i \in Q_{0}} \mathrm{Gr}_{e_{i}}\left(M_{i}\right)$ which is a projective homogeneous space for $G_{\boldsymbol{d}}$ of dimension $\sum_{i \in Q_{0}} e_{i}\left(d_{i}-e_{i}\right)$, namely $\operatorname{Gr}_{\boldsymbol{e}}(\boldsymbol{d}) \simeq G_{\boldsymbol{d}} / P_{\boldsymbol{e}}$ for a maximal parabolic $P_{\boldsymbol{e}} \subset G_{\boldsymbol{d}}$. We define $\mathrm{Gr}_{\boldsymbol{e}}^{Q}(\boldsymbol{d})$, the universal Grassmannian of $\boldsymbol{e}$-dimensional subrepresentations of $\boldsymbol{d}$-dimensional representations of $Q$, as the closed subvariety of $\operatorname{Gr}_{e}(d) \times R_{d}(Q)$ consisting of tuples

$$
\left(\left(U_{i} \subset M_{i}\right)_{i \in Q_{0}},\left(M_{\alpha}\right)_{\alpha \in Q_{1}}\right)
$$


such that $M_{\alpha}\left(U_{i}\right) \subset U_{j}$ for all arrows $(\alpha: i \rightarrow j) \in Q_{1}$. The group $G_{\boldsymbol{d}}$ acts on $\mathrm{Gr}_{\boldsymbol{e}}^{Q}(\boldsymbol{d})$ diagonally so that the projections $p_{1}: \mathrm{Gr}_{\boldsymbol{e}}^{Q}(\boldsymbol{d}) \rightarrow \mathrm{Gr}_{\boldsymbol{e}}(\boldsymbol{d})$ and $p_{2}: \mathrm{Gr}_{\boldsymbol{e}}^{Q}(\boldsymbol{d}) \rightarrow R_{\boldsymbol{d}}(Q)$ are $G_{\boldsymbol{d}}$-equivariant. In fact, the projection $p_{1}$ identifies $\operatorname{Gr}_{\boldsymbol{e}}^{Q}(\boldsymbol{d})$ as the total space of a homogeneous vector bundle over $\mathrm{Gr}_{e}(\boldsymbol{d})$ of rank

$$
\sum_{(\alpha: i \rightarrow j) \in Q_{1}}\left(d_{i} d_{j}+e_{i} e_{j}-e_{i} d_{j}\right)
$$

Indeed, for a point $\left(U_{i}\right)_{i=1}^{\# Q_{0}}$ in $\mathrm{Gr}_{\boldsymbol{e}}(\boldsymbol{d})$, we can choose complements $M_{i}=U_{i} \oplus V_{i}$ and identify the fiber of $p_{1}$ over $\left(U_{i}\right)_{i=1}^{\# Q_{0}}$ with

$$
\left(\left[\begin{array}{cc}
\operatorname{Hom}_{Q}\left(U_{i}, U_{j}\right) & \operatorname{Hom}_{Q}\left(V_{i}, U_{j}\right) \\
0 & \operatorname{Hom}_{Q}\left(V_{i}, V_{j}\right)
\end{array}\right] \subset \operatorname{Hom}_{Q}\left(M_{i}, M_{j}\right)\right)_{(\alpha: i \rightarrow j)} \subset R_{\boldsymbol{d}}(Q) .
$$

In particular, the universal Grassmannian $\operatorname{Gr}_{\boldsymbol{e}}^{Q}(\boldsymbol{d})$ is smooth and irreducible of dimension

$$
\operatorname{dim} \operatorname{Gr}_{\boldsymbol{e}}^{Q}(\boldsymbol{d})=\langle\boldsymbol{e}, \boldsymbol{d}-\boldsymbol{e}\rangle+\operatorname{dim} R_{\boldsymbol{d}}(Q) .
$$

The projection $p_{2}$ is proper, thus its image is a closed $G_{\boldsymbol{d}}$-stable subvariety of $R_{\boldsymbol{d}}$, consisting of representations admitting a subrepresentation of dimension vector $\boldsymbol{e}$.

We define the quiver Grassmannian $\operatorname{Gr}_{e}(M)=p_{2}^{-1}(M)$ as the fiber of $p_{2}$ over a point $M \in R_{\boldsymbol{d}}(Q)$; by definition, it parametrizes (more precisely, its closed points parametrize) $\boldsymbol{e}$-dimensional subrepresentations of the representation $M$.

Remark 2.1. Note that we have to view $\operatorname{Gr}_{e}(M)$ as a scheme; in particular, it might be nonreduced. For example, if $Q$ is the Kronecker quiver, $\boldsymbol{e}$ the isotropic root, and $M$ is a regular indecomposable representation of dimension vector $2 \boldsymbol{e}$, the quiver Grassmannian is Spec of the ring of dual numbers.

Recall that a representation $M$ is called exceptional if $\operatorname{Ext}_{Q}^{1}(M, M)=0$; thus, in view of (2-1), its orbit in $R_{\boldsymbol{d}}(Q)$ is open and dense.

Proposition 2.2. Let $M$ be an exceptional d-dimensional representation of $Q$. Then $\operatorname{Gr}_{\boldsymbol{e}}(M)$ is nonempty if $\operatorname{Ext}_{Q}^{1}(N, L)$ vanishes for generic $N$ of dimension vector $\boldsymbol{e}$ and generic $L$ of dimension vector $\boldsymbol{d}-\boldsymbol{e}$. In this case, $\mathrm{Gr}_{\boldsymbol{e}}(M)$ is smooth of dimension $\langle\boldsymbol{e}, \boldsymbol{d}-\boldsymbol{e}\rangle$, and for all $\boldsymbol{d}$-dimensional representations $N$, every irreducible component of $\mathrm{Gr}_{\boldsymbol{e}}(N)$ has at least dimension $\langle\boldsymbol{e}, \boldsymbol{d}-\boldsymbol{e}\rangle$.

Proof. The criterion for nonemptiness follows from [Schofield 1992, Theorem 3.3]. If $\operatorname{Gr}_{e}(M)$ is nonempty, $p_{2}$ is surjective with $\operatorname{Gr}_{e}(M)$ as its generic fiber. In particular, $\operatorname{Gr}_{\boldsymbol{e}}(M)$ is smooth of dimension $\langle\boldsymbol{e}, \boldsymbol{d}-\boldsymbol{e}\rangle$. For all other fibers, we obtain at least the desired estimate on dimensions of their irreducible components [Hartshorne 1977, Chapter II, Exercise 3.22 (b)]. 
We conclude this section by pointing out a useful isomorphism: let $U$ be a point of $\mathrm{Gr}_{\boldsymbol{e}}(M)$ and let $T_{U}\left(\mathrm{Gr}_{\boldsymbol{e}}(M)\right)$ denote the Zariski tangent space of $\mathrm{Gr}_{\boldsymbol{e}}(M)$ at $U$. As shown in [Schofield 1992; Caldero and Reineke 2008] we have the following scheme-theoretic description of the tangent space:

Lemma 2.3. For $U \in \mathrm{Gr}_{e}(M)$, we have $T_{U}\left(\operatorname{Gr}_{e}(M)\right) \simeq \operatorname{Hom}_{Q}(U, M / U)$.

Quotient construction of (universal) quiver Grassmannians and a stratification. We follow [Reineke 2008, Section 3.2]. Additionally to the choices before, fix vector spaces $N_{i}$ of dimension $e_{i}$ for $i \in Q_{0}$. We consider the universal variety $\operatorname{Hom}_{Q}(\boldsymbol{e}, \boldsymbol{d})$ of homomorphisms from an $\boldsymbol{e}$-dimensional to a $\boldsymbol{d}$-dimensional representation; explicitly, $\operatorname{Hom}_{Q}(\boldsymbol{e}, \boldsymbol{d})$ is the set of triples

$$
\left(\left(N_{\alpha}\right)_{\alpha \in Q_{1}},\left(f_{i}: N_{i} \rightarrow M_{i}\right)_{i \in Q_{0}},\left(M_{\alpha}\right)_{\alpha \in Q_{1}}\right) \in R_{\boldsymbol{e}} \times \prod_{i \in Q_{0}} \operatorname{Hom}\left(N_{i}, M_{i}\right) \times R_{\boldsymbol{d}}(Q)
$$

such that $f_{j} N_{\alpha}=M_{\alpha} f_{i}$ for all $(\alpha: i \rightarrow j) \in Q_{1}$. This is an affine variety defined by quadratic relations, namely by the vanishing of the individual entries of the matrices $f_{j} N_{\alpha}-M_{\alpha} f_{i}$, on which $G_{\boldsymbol{e}} \times G_{\boldsymbol{d}}$ acts naturally. On the open subset $\operatorname{Hom}_{Q}^{0}(\boldsymbol{e}, \boldsymbol{d})$ where all $f_{i}: N_{i} \rightarrow M_{i}$ are injective maps, the action of $G_{\boldsymbol{e}}$ is free. By construction, we have an isomorphism

$$
\operatorname{Hom}_{Q}^{0}(\boldsymbol{e}, \boldsymbol{d}) / G_{e} \simeq \operatorname{Gr}_{\boldsymbol{e}}^{Q}(\boldsymbol{d})
$$

which associates the orbit of a triple $\left(\left(N_{\alpha}\right),\left(f_{i}\right),\left(M_{\alpha}\right)\right)$ with the pair given by $\left.\left(f_{i}\left(N_{i}\right) \subset M_{i}\right)_{i \in Q_{0}},\left(M_{\alpha}\right)_{\alpha \in Q_{1}}\right)$. Indeed, the maps $N_{\alpha}$ are uniquely determined in this situation, and they can be reconstructed algebraically from $\left(f_{i}\right)$ and $\left(M_{\alpha}\right)$ (see [Reineke 2008, Lemma 3.5]).

Similarly to $\mathrm{Gr}_{\boldsymbol{e}}^{Q}(\boldsymbol{d})$, we have a projection $\tilde{p}_{2}: \operatorname{Hom}_{Q}^{0}(\boldsymbol{e}, \boldsymbol{d}) \rightarrow R_{\boldsymbol{d}}(Q)$ with fibers $\tilde{p}_{2}^{-1}(M)=\operatorname{Hom}_{Q}^{0}(\boldsymbol{e}, M)$, and we have a local version of the previous isomorphism:

$$
\operatorname{Hom}_{Q}^{0}(\boldsymbol{e}, M) / G_{\boldsymbol{e}}=\tilde{p}_{2}^{-1}(M) / G_{\boldsymbol{e}} \simeq \mathrm{Gr}_{\boldsymbol{e}}(M) .
$$

Note that the quotient map $\operatorname{Hom}_{Q}^{0}(\boldsymbol{e}, M) \rightarrow \mathrm{Gr}_{\boldsymbol{e}}(M)$ is locally trivial since it is induced by the quotient map

$$
\operatorname{Hom}_{Q}^{0}(\boldsymbol{e}, \boldsymbol{d})=\prod_{i \in Q_{0}} \operatorname{Hom}^{0}\left(N_{i}, M_{i}\right) \rightarrow \operatorname{Gr}_{\boldsymbol{e}}(\boldsymbol{d}),
$$

which can be trivialized over the standard open affine coverings of Grassmannians.

Let $p$ be the projection from $\operatorname{Hom}_{Q}^{0}(\boldsymbol{e}, M)$ to $R_{\boldsymbol{e}}(Q)$; its fiber over $N$ is the space $\operatorname{Hom}_{Q}^{0}(N, M)$ of injective maps. For each isomorphism class $[N]$ of representations of dimension vector $\boldsymbol{e}$, we can consider the subset $\mathscr{Y}_{[N]}$ of $\operatorname{Gr}_{\boldsymbol{e}}(M)$ corresponding under the previous isomorphism to $\left(p^{-1}\left(O_{N}\right)\right) / G_{e}$. Therefore it consists of all subrepresentations $U \in \operatorname{Gr}_{e}(M)$ which are isomorphic to $N$. 
Lemma 2.4. Each $\mathscr{S}_{[N]}$ is an irreducible locally closed subset of $\operatorname{Gr}_{\boldsymbol{e}}(M)$ of dimension $\operatorname{dim} \operatorname{Hom}_{Q}(N, M)-\operatorname{dim} \operatorname{End}_{Q}(N)$.

Proof. Irreducibility of $\mathscr{Y}_{[N]}$ follows from irreducibility of $\mathcal{O}_{N}$ by $G_{e}$-equivariance of $p$. Using the fact that the geometric quotient is closed and separating on $G_{e^{-}}$ stable subsets, induction over $\operatorname{dim} \mathscr{O}_{N}$ proves that all $\mathscr{S}_{[N]}$ are locally closed. The dimension is calculated as

$$
\operatorname{dim} \mathscr{Y}_{[N]}=\operatorname{dim} \mathscr{O}_{N}+\operatorname{dim} \operatorname{Hom}_{Q}^{0}(N, M)-\operatorname{dim} G_{e} .
$$

Degenerate flag varieties. In this subsection we recall the definition of the degenerate flag varieties following [Feigin 2010; 2011; Feigin and Finkelberg 2011]. Let $W$ be an $n$-dimensional vector space with a basis $w_{1}, \ldots, w_{n}$. We denote by $\mathrm{pr}_{k}: W \rightarrow W$ the projections along $w_{k}$ to the linear span of the remaining basis vectors, that is, $\operatorname{pr}_{k} \sum_{i=1}^{n} c_{i} w_{i}=\sum_{i \neq k} c_{i} w_{i}$.

Definition 2.5. The variety $\mathcal{F}_{n}^{a}$ is the set of collections of subspaces

$$
\left(V_{i} \in \mathrm{Gr}_{i}(W)\right)_{i=1}^{n-1}
$$

subject to the conditions $\mathrm{pr}_{i+1} V_{i} \subset V_{i+1}$ for all $i=1, \ldots, n-2$.

The variety $\mathcal{F}_{n}^{a}$ is called the complete degenerate flag variety. It enjoys the following properties:

- $\mathcal{F}_{n}^{a}$ is a singular irreducible projective algebraic variety of dimension $\left(\begin{array}{l}n \\ 2\end{array}\right)$.

- $\mathcal{F}_{n}^{a}$ is a flat degeneration of the classical complete flag variety $\mathrm{SL}_{n} / B$.

- $\mathcal{F}_{n}^{a}$ is a normal local complete intersection variety.

- $\mathcal{F}_{n}^{a}$ can be decomposed into a disjoint union of complex cells.

We add some comments on the last property. The number of cells (which is equal to the Euler characteristic of $\mathcal{F}_{n}^{a}$ ) is given by the $n$-th normalized median Genocchi number $h_{n}$; see for example [Feigin 2011, Section 3]. These numbers have several definitions. Here, we will use the following one: $h_{n}$ is the number of collections $\left(S_{1}, \ldots, S_{n-1}\right)$, where $S_{i} \subset\{1, \ldots, n\}$ subject to the conditions

$$
\# S_{i}=i, 1 \leq i \leq n-1 ; \quad S_{i} \subset S_{i+1} \cup\{i+1\}, 1 \leq i \leq n-2 .
$$

For $n=1,2,3,4,5$ the numbers $h_{n}$ are equal to $1,2,7,38,295$, respectively.

There exists a degeneration $\mathrm{SL}_{n}^{a}$ of the group $\mathrm{SL}_{n}$ acting on $\mathcal{F}_{n}^{a}$. Namely, the degenerate group $\mathrm{SL}_{n}^{a}$ is the semidirect product of the Borel subgroup $B$ of $\mathrm{SL}_{n}$ and a normal abelian subgroup $\mathbb{G}_{a}^{n(n-1) / 2}$, where $\mathbb{G}_{a}$ is the additive group of the field. The simplest way to describe the structure of the semidirect product is via the Lie algebra $\mathfrak{s l}_{n}^{a}$ of $\mathrm{SL}_{n}^{a}$. Namely, let $\mathfrak{b} \in \mathfrak{s l}_{n}$ be the Borel subalgebra of upper-triangular matrices and $\mathfrak{n}^{-}$be the nilpotent subalgebra of strictly lower-triangular matrices. 
Let $\left(\mathfrak{n}^{-}\right)^{a}$ be the abelian Lie algebra with underlying vector space $\mathfrak{n}^{-}$. Then $\mathfrak{n}^{-}$ carries a natural structure of $\mathfrak{b}$-module induced by the adjoint action on the quotient $\left(\mathfrak{n}^{-}\right)^{a} \simeq \mathfrak{s l}_{n} / \mathfrak{b}$. Then $\mathfrak{s l}_{n}^{a}=\mathfrak{b} \oplus\left(\mathfrak{n}^{-}\right)^{a}$, where $\left(\mathfrak{n}^{-}\right)^{a}$ is abelian ideal and $\mathfrak{b}$ acts on $\left(\mathfrak{n}^{-}\right)^{a}$ as described above. The group $\mathrm{SL}_{n}^{a}$ (the Lie group of $\mathfrak{s l}_{n}^{a}$ ) acts on the variety $\mathcal{F}_{n}^{a}$ with an open $\mathbb{G}_{a}^{n(n-1) / 2}$-orbit. We note that in contrast with the classical situation, the group $\mathrm{SL}_{n}^{a}$ acts on $\mathcal{F}_{n}^{a}$ with an infinite number of orbits.

For partial (parabolic) flag varieties of $\mathrm{SL}_{n}$ there exists a natural generalization of $\mathcal{F}_{n}^{a}$. Namely, consider an increasing collection $1 \leq d_{1}<\cdots<d_{s}<n$. In what follows we denote such a collection by $\boldsymbol{d}$. Let $\mathcal{F}_{\boldsymbol{d}}$ be the classical partial flag variety consisting of the collections $\left(V_{i}\right)_{i=1}^{s}, V_{i} \in \mathrm{Gr}_{d_{i}}(W)$ such that $V_{i} \subset V_{i+1}$.

Definition 2.6. The degenerate partial variety $\mathcal{F}_{\boldsymbol{d}}^{a}$ is the set of collections of subspaces $V_{i} \in \mathrm{Gr}_{d_{i}}(W)$ subject to the conditions $\mathrm{pr}_{d_{i}+1} \ldots \mathrm{pr}_{d_{i+1}} V_{i} \subset V_{i+1}$ for all $i=1, \ldots, s-1$.

We still have the following properties:

- $\mathcal{F}_{\boldsymbol{d}}^{a}$ is a singular irreducible projective algebraic variety.

- $\mathcal{F}_{\boldsymbol{d}}^{a}$ is a flat degeneration of $\mathcal{F}_{\boldsymbol{d}}$.

- $\mathcal{F}_{\boldsymbol{d}}^{a}$ is a normal local complete intersection variety.

- $\mathcal{F}_{\boldsymbol{d}}^{a}$ is acted upon by the group $\mathrm{SL}_{n}^{a}$ with an open $\mathbb{G}_{a}^{n(n-1) / 2}$-orbit.

Comparison between quiver Grassmannians and degenerate flag varieties. Let $Q$ be an equioriented quiver of type $A_{n}$. We order the vertices of $Q$ from 1 to $n$ in such a way that the arrows of $Q$ are of the form $i \rightarrow i+1$. Let $P_{i}$ and $I_{i}$, be the projective and injective representations, respectively, attached to the $i$-th vertex for $i=1, \ldots, n$. In particular, $\operatorname{dim} P_{i}=(0, \ldots, 0,1, \ldots, 1)$ with $i-1$ zeros and $\operatorname{dim} I_{i}=(1, \ldots, 1,0, \ldots, 0)$ with $n-i$ zeros.

We will use the following basis of $P_{i}$ and $I_{i}$ : for each $j=i, \ldots, n$ we fix nonzero elements $w_{i, j} \in\left(P_{j}\right)_{i}$ in such a way that $w_{i, j} \mapsto w_{i+1, j}$. Also, for $j=1, \ldots, i$, we fix nonzero elements $w_{i, j+1} \in\left(I_{j}\right)_{i}$ in such a way that $w_{i, j} \mapsto w_{i+1, j}$ unless $j=i+1$ and $w_{i, i+1} \mapsto 0$.

Let $A$ be the path algebra $\mathbb{C} Q$. Viewed as a representation of $Q, A$ is isomorphic to the direct sum $\bigoplus_{i=1}^{n} P_{i}$. In particular, $\operatorname{dim} A=(1,2, \ldots, n)$. The linear dual $A^{*}$ is isomorphic to the direct sum of injective representations $\bigoplus_{i=1}^{n} I_{i}$.

Proposition 2.7. The quiver Grassmannian $\operatorname{Gr}_{\operatorname{dim} A}\left(A \oplus A^{*}\right)$ is isomorphic to the degenerate flag variety $\mathcal{F}_{n+1}^{a}$ of $\mathfrak{s l}_{n+1}$.

Proof. Consider $A \oplus A^{*}=\bigoplus_{i=1}^{n}\left(P_{i} \oplus I_{i}\right)$ as a representation of $Q$. Let $W_{j}$ be the space attached to the $j$-th vertex, that is, $A \oplus A^{*}=\left(W_{1}, \ldots, W_{n}\right)$. First, we note that $\operatorname{dim} W_{j}=n+1$ for all $j$. Second, we fix an $(n+1)$-dimensional vector space $W$ with a basis $w_{1}, \ldots, w_{n+1}$. We identify all $W_{j}$ with $W$ by sending $w_{i, j}$ to $w_{j}$. 
Then the maps $W_{j} \rightarrow W_{j+1}$ coincide with $\mathrm{pr}_{j+1}$. Now our proposition follows from the equality $\operatorname{dim} A=(1,2, \ldots, n)$.

The coefficient quiver of the representation $A \oplus A^{*}$ is given by $(n=4)$ :

$$
\begin{aligned}
& w_{1,5} \rightarrow w_{2,5} \rightarrow w_{3,5} \rightarrow w_{4,5} \\
& w_{1,4} \rightarrow w_{2,4} \rightarrow w_{3,4} \quad w_{4,4} \\
& w_{1,3} \rightarrow w_{2,3} \quad w_{3,3} \rightarrow w_{4,3} \\
& w_{1,2} \quad w_{2,2} \rightarrow w_{3,2} \rightarrow w_{4,2} \\
& w_{1,1} \rightarrow w_{2,1} \rightarrow w_{3,1} \rightarrow w_{4,1}
\end{aligned}
$$

Remark 2.8. We note that the classical $\mathrm{SL}_{n+1}$ flag variety has a similar realization. Namely, let $\tilde{M}$ be the representation of $Q$ isomorphic to the direct sum of $n+1$ copies of $P_{1}, \operatorname{so} \operatorname{dim} \tilde{M}=\operatorname{dim}\left(A \oplus A^{*}\right)$. Then the classical flag variety $\mathrm{SL}_{n+1} / B$ is isomorphic to the quiver Grassmannian $\operatorname{Gr}_{\operatorname{dim} A} \tilde{M}$. The $Q$-representation $\tilde{M}$ can be visualized as $(n=4)$

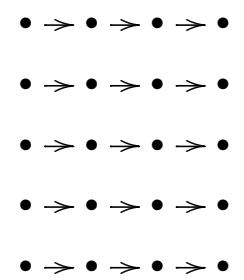

We can easily generalize Proposition 2.7 to degenerate partial flag varieties:

Suppose we are given a sequence $\boldsymbol{d}=\left(0=d_{0}<d_{1}<d_{2}<\ldots<d_{s}<d_{s+1}=n+1\right)$. Then we define

$$
P=\bigoplus_{i=1}^{s} P_{i}^{d_{i}-d_{i-1}} \quad \text { and } \quad I=\bigoplus_{i=1}^{s} I_{i}^{d_{i+1}-d_{i}}
$$

as representations of an equioriented quiver of type $A_{s}$.

Proposition 2.9. The quiver Grassmannian $\mathrm{Gr}_{\operatorname{dim} P}(P \oplus I)$ is isomorphic to the degenerate partial flag variety $\mathcal{F}_{\boldsymbol{d}}^{a}$ of $\mathfrak{s l}_{n+1}$.

Proof. The dimension vector of $P \oplus I$ is given by $(n+1, \ldots, n+1)$ and the dimension vector of $P$ equals $\left(d_{1}, \ldots, d_{s}\right)$. Now let us identify the spaces $(P \oplus I)_{j}$ with $W$ as in the proof of Proposition 2.7. Then the map

$$
(P \oplus I)_{j} \rightarrow(P \oplus I)_{j+1}
$$

corresponding to the arrow $j \rightarrow j+1$ coincides with $\operatorname{pr}_{d_{j}+1}, \ldots, \mathrm{pr}_{d_{j+1}}$, which proves the proposition. 


\section{A class of well-behaved quiver Grassmannians}

Geometric properties. From now on, let $Q$ be a Dynkin quiver. Then $G_{\boldsymbol{d}}$ acts with finitely many orbits on $R_{\boldsymbol{d}}(Q)$ for every $\boldsymbol{d}$; in particular, for every $\boldsymbol{d} \in \mathbb{N} Q_{0}$, there exists a unique (up to isomorphism) exceptional representation of this dimension vector.

The subsets $\mathscr{Y}_{[N]}$ defined just before Lemma 2.4 then define a finite stratification of each quiver Grassmannian $\operatorname{Gr}_{e}(M)$ according to isomorphism type of the subrepresentation $N \subset M$.

Proposition 3.1. Assume that $X$ and $Y$ are exceptional representations of $Q$ such that $\operatorname{Ext}_{Q}^{1}(X, Y)=0$. Define $M=X \oplus Y$ and $\boldsymbol{e}=\operatorname{dim} X, \boldsymbol{d}=\operatorname{dim}(X \oplus Y)$. Then:

(i) $\operatorname{dim} \mathrm{Gr}_{\boldsymbol{e}}(M)=\langle\boldsymbol{e}, \boldsymbol{d}-\boldsymbol{e}\rangle$.

(ii) The variety $\mathrm{Gr}_{e}(M)$ is reduced, irreducible and rational.

(iii) $\mathrm{Gr}_{e}(M)$ is a locally complete intersection scheme.

Proof. The representation $X$ obviously embeds into $M$; thus

$$
\begin{aligned}
& \operatorname{dim} \operatorname{Gr}_{\boldsymbol{e}}(M) \geq \operatorname{dim} \mathscr{Y}_{[X]}=\operatorname{dim} \operatorname{Hom}_{Q}(X, M)-\operatorname{dim} \operatorname{End}_{Q}(X) \\
& =\operatorname{dim} \operatorname{Hom}_{Q}(X, Y) \text {. }
\end{aligned}
$$

The tangent space to any point $U \in \mathscr{T}_{[X]}$ has dimension $\operatorname{dim}_{\operatorname{Hom}_{Q}}(X, Y)$ too, thus $\overline{\mathscr{Y}_{[X]}}$ is reduced. Moreover, a generic embedding of $X$ into $X \oplus Y$ is of the form $\left[\operatorname{id}_{X}, f\right]$ for a map $f \in \operatorname{Hom}_{Q}(X, Y)$, and this identifies an open subset isomorphic to $\operatorname{Hom}_{Q}(X, Y)$ of $\mathscr{Y}_{[X]}$, proving rationality of $\overline{\mathscr{Y}_{[X]}}$. Now suppose $N$ embeds into $M=X \oplus Y$ and $\operatorname{dim} N=\boldsymbol{e}$. Then $\operatorname{Ext}_{Q}^{1}(N, Y)=0$ since $\operatorname{Ext}_{Q}^{1}(X \oplus Y, Y)=0$ by assumption, and thus $\operatorname{dim}_{\operatorname{Hom}_{Q}}(N, Y)=\langle\boldsymbol{e}, \boldsymbol{d}-\boldsymbol{e}\rangle=\operatorname{dim}_{\operatorname{Hom}_{Q}}(X, Y)$. Therefore,

$$
\begin{aligned}
\operatorname{dim} \mathscr{S}_{[N]} & =\operatorname{dim} \operatorname{Hom}_{Q}(N, X)-\operatorname{dim} \operatorname{Hom}_{Q}(N, N)+\operatorname{dim} \operatorname{Hom}_{Q}(X, Y) \\
& \leq \operatorname{dim} \operatorname{Hom}_{Q}(X, Y),
\end{aligned}
$$

which proves that $\operatorname{dim} \operatorname{Gr}_{\boldsymbol{e}}(M)=\operatorname{dim} \operatorname{Hom}_{Q}(X, Y)=\langle\boldsymbol{e}, \boldsymbol{d}-\boldsymbol{e}\rangle$, and that the closure of $\mathscr{Y}_{[X]}$ is an irreducible component of $\operatorname{Gr}_{\boldsymbol{e}}(M)$. Conversely, suppose that an irreducible component $C$ of $\operatorname{Gr}_{e}(M)$ is given. Then $C$ is necessarily the closure of some stratum $\mathscr{Y}_{[N]}$, and the dimension of $C$ equals $\langle\boldsymbol{e}, \boldsymbol{d}-\boldsymbol{e}\rangle=\operatorname{dim}_{\operatorname{Hom}}(X, Y)$ by Proposition 2.2. By the dimension estimate above, we conclude that

$$
\operatorname{dim} \operatorname{Hom}_{Q}(N, X)=\operatorname{dim} \operatorname{Hom}_{Q}(N, N) .
$$

By [Bongartz 1996, Theorem 2.4], this yields an embedding $N \subset X$, and thus $N=X$ by equality of dimensions. Therefore, $\operatorname{Gr}_{e}(M)$ equals the closure of the stratum $\mathscr{Y}_{[X]}$, thus it is irreducible, reduced and rational. The dimension of $\operatorname{Hom}_{Q}^{0}(\boldsymbol{e}, M)$ 
equals $\langle\boldsymbol{e}, \boldsymbol{d}-\boldsymbol{e}\rangle+\operatorname{dim} G_{\boldsymbol{e}}$, thus its codimension in $R_{\boldsymbol{e}}(Q) \times \operatorname{Hom}_{Q}^{0}(\boldsymbol{e}, \boldsymbol{d})$ equals

$$
\operatorname{dim} R_{\boldsymbol{e}}(Q)+\sum_{i} e_{i} d_{i}-\langle\boldsymbol{e}, \boldsymbol{d}-\boldsymbol{e}\rangle-\operatorname{dim} G_{\boldsymbol{e}}=\sum_{(\alpha: i \rightarrow j) \in Q_{1}} e_{i} d_{j} .
$$

But this value is exactly the number of equations defining $\operatorname{Hom}_{Q}(\boldsymbol{e}, M)$. Thus $\operatorname{Hom}_{Q}^{0}(\boldsymbol{e}, M)$ is locally a complete intersection. The $\operatorname{map}_{\operatorname{Hom}^{0}}^{0}(\boldsymbol{e}, M) \rightarrow \operatorname{Gr}_{\boldsymbol{e}}(M)$ is locally trivial with smooth fiber $G_{\boldsymbol{e}}$, hence the last statement follows.

On a quiver Grassmannian $\operatorname{Gr}_{e}(M)$, the automorphism group $\operatorname{Aut}_{Q}(M)$ acts algebraically. In the present situation, this implies that the group

$$
G=\left[\begin{array}{cc}
\operatorname{Aut}_{Q}(X) & 0 \\
\operatorname{Hom}_{Q}(X, Y) & \operatorname{Aut}_{Q}(Y)
\end{array}\right]
$$

acts on $\mathrm{Gr}_{e}(X \oplus Y)$.

Flat degeneration. Now let $\tilde{M}$ be the unique (up to isomorphism) exceptional representation of the same dimension vector as $M$. By Proposition 2.2, we also have $\operatorname{dim} \operatorname{Gr}_{\boldsymbol{e}}(\tilde{M})=\langle\boldsymbol{e}, \boldsymbol{d}-\boldsymbol{e}\rangle$. It is thus reasonable to ask for good properties of the degeneration from $\mathrm{Gr}_{e}(\tilde{M})$ to $\mathrm{Gr}_{e}(M)$.

Theorem 3.2. Under the previous hypotheses, the quiver Grassmannian $\mathrm{Gr}_{e}(M)$ is a flat degeneration of $\operatorname{Gr}_{\boldsymbol{e}}(\tilde{M})$.

Proof. Let $Y$ be the open subset of $R_{\boldsymbol{d}}(Q)$ consisting of all representations $Z$ whose orbit closure $\overline{\mathrm{O}_{Z}}$ contains the orbit $\mathrm{O}_{M}$; in particular, $Y$ contains $\widetilde{O}_{\tilde{M}}$. We consider the diagram

$$
\mathrm{Gr}_{\boldsymbol{e}}(\boldsymbol{d}) \stackrel{p_{1}}{\longleftarrow} \mathrm{Gr}_{\boldsymbol{e}}^{Q}(\boldsymbol{d}) \stackrel{p_{2}}{\longrightarrow} R_{\boldsymbol{d}}(Q)
$$

of the previous section. In particular, we consider the restriction $q: \widetilde{Y} \rightarrow Y$ of $p_{2}$ to $\widetilde{Y}=p_{2}^{-1}(Y)$. This is a proper morphism (since $p_{2}$ is so) between two smooth and irreducible varieties (since they are open subsets of the smooth varieties $R_{d}(Q)$ and $\operatorname{Gr}_{\boldsymbol{e}}^{Q}(\boldsymbol{d})$, respectively). The general fiber of $q$ is $\operatorname{Gr}_{\boldsymbol{e}}(\tilde{M})$, since the orbit of $\widetilde{M}$, being exceptional, is open in $Y$, and the special fiber of $q$ is $\operatorname{Gr}_{e}(M)$, since the orbit of $M$ is closed in $Y$ by definition. By semicontinuity, all fibers of $q$ have the same dimension $\langle\boldsymbol{e}, \boldsymbol{d}-\boldsymbol{e}\rangle$. By [Matsumura 1989, Corollary to Theorem 23.1], a proper morphism between smooth and irreducible varieties with constant fiber dimension is already flat.

Remark 3.3. Theorem 3.2 generalizes Proposition 3.15 of [Feigin 2010], where the flatness of the degeneration $\mathcal{F}_{n} \rightarrow \mathcal{F}_{n}^{a}$ was proved using complicated combinatorial tools. (See also the sections on degenerate flag varieties in the present paper, pages 172-174.) 
Note that the degeneration from $\tilde{M}$ to $M$ in $R_{d}(Q)$ can be realized along a one-parameter subgroup of $G_{\boldsymbol{d}}$ in the following way:

Lemma 3.4. Under the previous hypotheses, there exists a short exact sequence

$$
0 \rightarrow X \rightarrow \tilde{M} \rightarrow Y \rightarrow 0 .
$$

Proof. By [Schofield 1992, Theorem 3.3], a generic representation $Z$ of dimension vector $\boldsymbol{d}$ admits a subrepresentation of dimension vector $\boldsymbol{e}$ if $\operatorname{Ext}_{Q}^{1}(N, L)$ vanishes for generic $N$ of dimension vector $\boldsymbol{e}$ and generic $L$ of dimension vector $\boldsymbol{d}-\boldsymbol{e}$. In the present case, these generic representations are $Z=\tilde{M}, N=X$ and $L=Y$, and the lemma follows.

This lemma implies that $\widetilde{M}$ can be written, up to isomorphism, in the following form

$$
\widetilde{M}_{\alpha}=\left[\begin{array}{cc}
X_{\alpha} & \zeta_{\alpha} \\
0 & Y_{\alpha}
\end{array}\right]
$$

for all $\alpha \in Q_{1}$. Conjugating with the one-parameter subgroup

$$
\left(\left[\begin{array}{cc}
t \cdot \operatorname{id} X_{i} & 0 \\
0 & \operatorname{id} Y_{i}
\end{array}\right]\right)_{i \in Q_{0}}
$$

of $G_{\boldsymbol{d}}$ and passing to the limit $t=0$, we arrive at the desired degeneration.

Since $Q$ is a Dynkin quiver, the isomorphism classes of indecomposable representations of $Q$ are parametrized by the positive roots $\Phi^{+}$of the corresponding root system. We view $\Phi^{+}$as a subset of $\mathbb{N} Q_{0}$ by identifying the simple root $\alpha_{i}$ with the vector having 1 at the $i$-th place and zeros everywhere else. Denote by $V_{\alpha}$ the indecomposable representation corresponding to $\alpha \in \Phi^{+}$; more precisely, $\operatorname{dim} V_{\alpha}=\alpha$. Using this parametrization of the indecomposables and the AuslanderReiten quiver of $Q$, we can actually construct $\widetilde{M}$ explicitly from $X$ and $Y$ (or, more precisely, from their decompositions into indecomposables), using the algorithm of [Reineke 2001, Section 3].

\section{The group action and normality}

In this section we put $X=P$ and $Y=I$, where $P$ and $I$ are projective and injective representations of a Dynkin quiver $Q$. We consider the group

$$
G=\left[\begin{array}{cc}
\operatorname{Aut}_{Q}(P) & 0 \\
\operatorname{Hom}_{Q}(P, I) & \operatorname{Aut}_{Q}(I)
\end{array}\right] .
$$

Theorem 4.1. The group $G$ acts on $\operatorname{Gr}_{\operatorname{dim} P}(P \oplus I)$ with finitely many orbits, parametrized by pairs $\left(\left[Q_{P}\right],\left[N_{I}\right]\right)$ of isomorphism classes such that $Q_{P}$ is a quotient of $P, N_{I}$ is a subrepresentation of $I$, and $Q_{P}$ and $N_{I}$ have the same dimension vector. 
Proof. Suppose $N$ is a subrepresentation of $P \oplus I$ of dimension vector $\operatorname{dim} N=$ $\operatorname{dim} P$, and denote by $\iota: N \rightarrow P \oplus I$ the embedding. Define $N_{I}=N \cap I$ and $N_{P}=N /(N \cap I)$. Then $N_{P} \simeq(N+I) / I$ embeds into $(P \oplus I) / I \simeq P$, thus $N_{P}$ is projective since $\operatorname{rep}(Q)$ is hereditary. Therefore, the short exact sequence

$$
0 \rightarrow N_{I} \rightarrow N \rightarrow N_{P} \rightarrow 0
$$

splits. We thus have a retraction $r: N_{P} \rightarrow N$ such that $N$ is the direct sum of $N_{I}$ and $r\left(N_{P}\right)$ and $N_{I}$ embeds into the component $I$ of $P \oplus I$ under $\iota$. Without loss of generality, we can thus write the embedding of $N$ into $P \oplus I$ as

$$
\iota=\left[\begin{array}{cc}
\iota_{P} & 0 \\
f & \iota_{I}
\end{array}\right]: N_{P} \oplus N_{I} \rightarrow P \oplus I
$$

for $\iota_{P}$ (resp. $\left.\iota_{I}\right)$ an embedding of $N_{P}$ (resp. $N_{I}$ ) into $P$ (resp. $I$ ), and $f: N_{P} \rightarrow I$. Since $I$ is injective, the map $f$ factors through $\iota_{P}$, yielding a map $x: P \rightarrow I$ such that $x \iota_{P}=f$. We can then conjugate $\iota$ with

$$
\left[\begin{array}{cc}
1 & 0 \\
-x & 1
\end{array}\right] \in G
$$

We have thus proved that each $G$-orbit in $\mathrm{Gr}_{\operatorname{dim}_{P}}(P \oplus I)$ contains an embedding of the form

$$
\left[\begin{array}{cc}
\iota_{P} & 0 \\
0 & \iota_{I}
\end{array}\right]: N_{P} \oplus N_{I} \rightarrow P \oplus I
$$

such that $N_{I}$ is a subrepresentation of $I$, the representation $Q_{P}=P / N_{P}$ is a quotient of $P$, and their dimension vectors obviously add up to $\operatorname{dim} P$. We now have to show that the isomorphism classes of such $Q_{P}$ and $N_{I}$ already characterize the corresponding $G$-orbit in $\mathrm{Gr}_{\operatorname{dim} P}(P \oplus I)$. To do this, suppose we are given two such embeddings,

$$
\left[\begin{array}{cc}
\iota_{P} & 0 \\
0 & \iota_{I}
\end{array}\right]: N_{P} \oplus N_{I} \rightarrow P \oplus I \quad \text { and } \quad\left[\begin{array}{cc}
\iota_{P}^{\prime} & 0 \\
0 & \iota_{I}^{\prime}
\end{array}\right]: N_{P}^{\prime} \oplus N_{I}^{\prime} \rightarrow P \oplus I
$$

such that the cokernels $Q_{P}$ and $Q_{P}^{\prime}$ of $\iota_{P}$ and $\iota_{P}^{\prime}$, respectively, are isomorphic, and such that $N_{I}$ and $N_{I}^{\prime}$ are isomorphic. By [Reineke 2008, Lemma 6.3], an arbitrary isomorphism $\psi_{I}: N_{I} \rightarrow N_{I}^{\prime}$ lifts to an automorphism $\varphi_{I}$ of $I$, such that $\varphi_{I} \iota_{I}=\iota_{I}^{\prime} \psi_{I}$. By the obvious dual version of the same lemma, an arbitrary isomorphism $\xi_{P}: Q_{P} \rightarrow Q_{P}^{\prime}$ lifts to an automorphism $\varphi_{P}$ of $P$, which in turn induces an isomorphism $\psi_{P}: N_{P} \rightarrow N_{P}^{\prime}$ such that $\varphi_{P} \iota_{P}=\iota_{P}^{\prime} \psi_{P}$. This proves that the two embeddings above are conjugate under $G$. Finally, given representations $Q_{P}$ and $N_{I}$ as above, we can define $N_{P}$ as the kernel of the quotient map and get an embedding as above. 
Remark 4.2. We can obtain an explicit parametrization of the orbits by writing

$$
P=\bigoplus_{i \in Q_{0}} P_{i}^{a_{i}} \quad \text { and } \quad I=\bigoplus_{i \in Q_{0}} I_{i}^{b_{i}} .
$$

By [Reineke 2008, Lemma 4.1] and its obvious dual version, we have:

- A representation $N_{I}$ embeds into $I$ if and only if $\operatorname{dim}_{\operatorname{Hom}}\left(S_{i}, N_{I}\right) \leq b_{i}$ for all $i \in Q_{0}$.

- A representation $Q_{P}$ is a quotient of $P$ if and only if $\operatorname{dim}_{\operatorname{Hom}}\left(Q_{P}, S_{i}\right) \leq a_{i}$ for all $i \in Q_{0}$.

The previous result establishes a finite decomposition of the quiver Grassmannians into orbits. In particular the tangent space is equidimensional along every such orbit. The following example shows that in general such orbits are not cells.

Example 4.3. Let

$$
Q:=\quad 1 \longrightarrow 2 \longrightarrow \nearrow^{3}
$$

be a Dynkin quiver of type $D_{4}$. The quiver Grassmannian $\operatorname{Gr}_{(1211)}\left(I_{3} \oplus I_{4}\right)$ is isomorphic to $\mathbb{P}^{1}$, with the points 0 and $\infty$ corresponding to two decomposable representations, whereas all points in $\mathbb{P}^{1} \backslash\{0, \infty\}$, which is obviously not a cell, correspond to subrepresentations which are isomorphic to the indecomposable representation of dimension vector (1211).

We note the following generalization of the tautological bundles

$$
U_{i}=\left\{(U, x) \in \operatorname{Gr}_{e}(X) \times X_{i}: x \in U_{i}\right\} \quad \text { on } \operatorname{Gr}_{e}(X):
$$

Given a projective representation $P$, the trivial vector bundle $\operatorname{Hom}_{Q}(P, X)$ on $\mathrm{Gr}_{e}(X)$ admits the subbundle

$$
\mathscr{V}_{P}=\left\{(U, \alpha) \in \operatorname{Gr}_{\boldsymbol{e}}(X) \times \operatorname{Hom}_{Q}(P, X): \alpha(P) \subset U\right\} .
$$

We then have $\mathscr{V}_{P} \simeq \bigoplus_{i \in Q_{0}} \mathscr{V}_{i}^{m_{i}}$ if $P \simeq \bigoplus_{i \in Q_{0}} P_{i}^{m_{i}}$. Dually, given an injective representation $I$, the trivial vector bundle $\operatorname{Hom}_{Q}(X, I)$ admits the subbundle

$$
\mathscr{V}_{I}=\left\{(U, \beta) \in \operatorname{Gr}_{e}(X) \times \operatorname{Hom}_{Q}(X, I): \beta(U)=0\right\} .
$$

We then have $\mathscr{V}_{I} \simeq \bigoplus_{i \in Q_{0}}\left(\mathscr{V}_{i}^{*}\right)^{m_{i}}$ if $I \simeq \bigoplus_{i \in Q_{0}} I_{i}^{m_{i}}$.

Given a decomposition of the dimension vector $\operatorname{dim} P=\boldsymbol{e}=\boldsymbol{f}+\boldsymbol{g}$, recall the subvariety $\mathscr{Y}_{f}(P \oplus I) \subset \mathrm{Gr}_{\boldsymbol{e}}(P \oplus I)$ consisting of all representations $N$ such that $\operatorname{dim} N \cap I=f$ and $\operatorname{dim} \pi(N)=\boldsymbol{g}$, where $\pi: P \oplus I \rightarrow P$ is the natural projection. We have a natural surjective map $\zeta: \operatorname{Gr}_{f, g}(P \oplus I) \rightarrow \operatorname{Gr}_{g}(P) \times \operatorname{Gr}_{f}(I)$. We note that since $P$ is projective, all the points of $\operatorname{Gr}_{g}(P)$ are isomorphic as representations of $Q$. 
Also, since $I$ is injective, for any two points $M_{1}, M_{2} \in \mathrm{Gr}_{f}(I)$ the representations $I / M_{1}$ and $I / M_{2}$ of $Q$ are isomorphic. Therefore, the dimension of the vector space $\operatorname{Hom}_{Q}\left(N_{P}, I / N_{I}\right)$ is independent of the points $N_{P} \in \operatorname{Gr}_{g}(P)$ and $N_{I} \in \operatorname{Gr}_{f}(I)$. We denote this dimension by $D$.

Proposition 4.4. The map $\zeta$ is a D-dimensional vector bundle (in the Zariski topology).

Proof. Associated to $N_{P}$ and $N_{I}$, we have exact sequences

$$
0 \rightarrow N_{P} \rightarrow P \rightarrow Q_{P} \rightarrow 0 \quad \text { and } \quad 0 \rightarrow N_{I} \rightarrow I \rightarrow Q_{I} \rightarrow 0 .
$$

These induce the following commutative diagram with exact rows and columns, where the final zeroes arise from projectivity of $N_{P}$ and injectivity of $Q_{I}$ and we abbreviate $\operatorname{Hom}_{Q}\left({ }_{-},{ }_{-}\right)$by $\left({ }_{-},{ }_{-}\right)$:

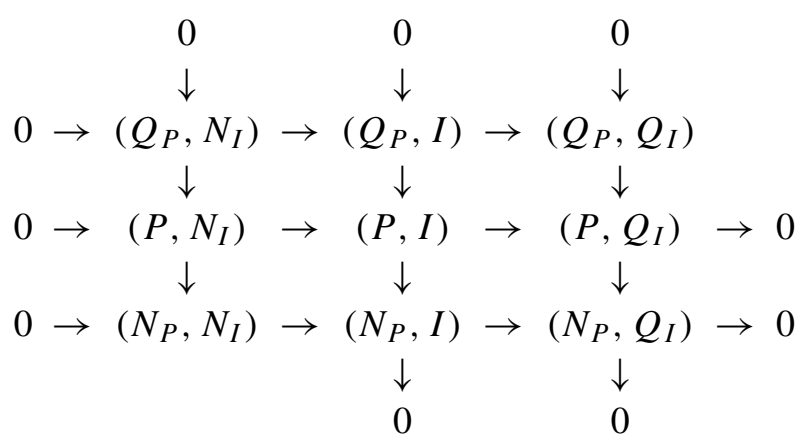

This diagram yields an isomorphism

$$
\operatorname{Hom}_{Q}\left(N_{P}, Q_{I}\right) \simeq \operatorname{Hom}_{Q}(P, I) /\left(\operatorname{Hom}_{Q}\left(P, N_{I}\right)+\operatorname{Hom}_{Q}\left(Q_{P}, I\right)\right) .
$$

Pulling back the tautological bundles constructed above via the projections

$$
\mathrm{Gr}_{g}(P) \stackrel{\mathrm{pr}_{1}}{\leftarrow} \mathrm{Gr}_{g}(P) \times \mathrm{Gr}_{f}(I) \stackrel{\mathrm{pr}_{2}}{\rightarrow} \mathrm{Gr}_{f}(I),
$$

we get subbundles $\operatorname{pr}_{2}^{* \mathscr{V}} \mathcal{V}_{P}$ and $\operatorname{pr}_{1}^{* \mathscr{V}} \mathscr{V}_{I}$ of the trivial bundle $\operatorname{Hom}_{Q}(P, I)$ on $\operatorname{Gr}_{g}(P) \times$ $\operatorname{Gr}_{f}(I)$. By the above isomorphism, the quotient bundle

$$
\operatorname{Hom}_{Q}(P, I) /\left(\operatorname{pr}_{1}^{*} \mathscr{V}_{P}+\operatorname{pr}_{2}^{*} \mathscr{V}_{I}\right)
$$

identifies with the fibration $\zeta: \mathscr{S}_{f}(P \oplus I) \rightarrow \operatorname{Gr}_{g}(P) \times \mathrm{Gr}_{f}(I)$, proving the Zariski local triviality of the latter.

The methods established in the two previous proofs now allow us to prove normality of the quiver Grassmannians.

Theorem 4.5. The quiver Grassmannian $\mathrm{Gr}_{e}(P \oplus I)$ is a normal variety. 
Proof. We already know that $\operatorname{Gr}_{\boldsymbol{e}}(P \oplus I)$ is locally a complete intersection, thus normality is proved once we know that $\operatorname{Gr}_{e}(P \oplus I)$ is regular in codimension 1 . By the proof of Theorem 4.1, we know that a subrepresentation $N$ of $P \oplus I$ of dimension vector $\operatorname{dim} P$ is of the form $N=N_{P} \oplus N_{I}$, with exact sequences

$$
0 \rightarrow N_{P} \rightarrow P \rightarrow Q_{P} \rightarrow 0 \text { and } 0 \rightarrow N_{I} \rightarrow I \rightarrow Q_{I} \rightarrow 0
$$

such that $N_{I}$ and $Q_{P}$ are of the same dimension vector $\boldsymbol{f}$. By the tangent space formula, $N$ defines a singular point of $\mathrm{Gr}_{e}(P \oplus I)$ if and only if

$$
\operatorname{Ext}_{Q}^{1}\left(N_{P} \oplus N_{I}, Q_{P} \oplus Q_{I}\right)=\operatorname{Ext}_{Q}^{1}\left(N_{I}, Q_{P}\right)
$$

is nonzero. In particular, singularity of the point $N$ only depends on the isomorphism types of $N_{I}=N \cap I$ and $Q_{P}=(P \oplus I) /(N+I)$. Consider the locally closed subset $Z$ of $\operatorname{Gr}_{e}(P \oplus I)$ consisting of subrepresentations $N^{\prime}$ such that $N^{\prime} \cap I \simeq N_{I}$ and $(P \oplus I) /\left(N^{\prime}+I\right) \simeq Q_{P}$; thus $Z \subset \mathscr{Y}_{f}$. The vector bundle $\zeta: \mathscr{S}_{f} \rightarrow \operatorname{Gr}_{f}(I) \times$ $\mathrm{Gr}_{\boldsymbol{e}-f}(P)$ of the previous proposition restricts to a vector bundle

$$
\zeta: Z \rightarrow Z_{I} \times Z_{P}
$$

where $Z_{I}=\mathscr{Y}_{\left[N_{I}\right]} \subset \mathrm{Gr}_{f}(I)$ consists of subrepresentations isomorphic to $N_{I}$, and $Z_{P} \subset \mathrm{Gr}_{e-f}(P)$ consists of subrepresentations with quotient isomorphic to $Q_{P}$. By the dimension formula for the strata $\mathscr{S}_{\left[N_{I}\right]}$, the codimension of $Z_{I}$ in $\mathrm{Gr}_{f}(I)$ equals $\operatorname{dim} \operatorname{Ext}_{Q}^{1}\left(N_{I}, N_{I}\right)$; dually, the codimension of $Z_{P}$ in $\operatorname{Gr}_{\boldsymbol{e}-\boldsymbol{f}}(P)$ equals $\operatorname{dim} \operatorname{Ext}_{Q}^{1}\left(Q_{P}, Q_{P}\right)$. Since the rank of the bundle $\zeta$ is $\operatorname{dim} \operatorname{Hom}_{Q}\left(N_{P}, Q_{I}\right)$, we have

$$
\begin{aligned}
& \operatorname{dim} \operatorname{Gr}_{\boldsymbol{e}}(P \oplus I)-\operatorname{dim} \zeta^{-1}\left(Z_{I} \times Z_{P}\right) \\
&=\operatorname{dim} \operatorname{Gr}_{\boldsymbol{e}}(P \oplus I)-\operatorname{dim} \operatorname{Hom}_{Q}\left(N_{P}, Q_{I}\right)-\left(\operatorname{dim}_{\left.\operatorname{Gr}_{\boldsymbol{f}}(I)-\operatorname{dim} \operatorname{Ext}_{Q}^{1}\left(N_{I}, N_{I}\right)\right)}\right.-\left(\operatorname{dim} \operatorname{Gr}_{\boldsymbol{e}-\boldsymbol{f}}(P)-\operatorname{dim} \operatorname{Ext}_{Q}^{1}\left(Q_{P}, Q_{P}\right)\right) \\
&=\langle\boldsymbol{e}, \boldsymbol{d}\rangle-\langle\boldsymbol{e}-\boldsymbol{f}, \boldsymbol{d}-\boldsymbol{f}\rangle-\langle\boldsymbol{f}, \boldsymbol{d}-\boldsymbol{f}\rangle-\langle\boldsymbol{e}-\boldsymbol{f}, \boldsymbol{f}\rangle \\
& \quad+\operatorname{dim}_{\operatorname{Ext}_{Q}\left(N_{I}, N_{I}\right)+\operatorname{dim} \operatorname{Ext}_{Q}^{1}\left(Q_{P}, Q_{P}\right)} \\
&=\langle\boldsymbol{f}, \boldsymbol{f}\rangle+\operatorname{dim} \operatorname{Ext}_{Q}^{1}\left(N_{I}, N_{I}\right)+\operatorname{dim} \operatorname{Ext}_{Q}^{1}\left(Q_{P}, Q_{P}\right)
\end{aligned}
$$

for the codimension of $Z$ in $\operatorname{Gr}_{e}(P \oplus I)$. Assume that this codimension equals 1. Since the Euler form ( $Q$ being Dynkin) is positive definite, the summand $\langle\boldsymbol{f}, \boldsymbol{f}\rangle$ is nonnegative. If it equals 0 , then $\boldsymbol{f}$ equals 0 , and $N_{I}$ and $Q_{P}$ are just the zero representations, a contradiction to the assumption $\operatorname{Ext}_{Q}^{1}\left(N_{I}, Q_{P}\right) \neq 0$. Thus $\langle\boldsymbol{f}, \boldsymbol{f}\rangle=1$ and both other summands are zero, thus $N_{I}$ and $Q_{P}$ are both isomorphic to the exceptional representation of dimension vector $f$. But this implies vanishing of $\operatorname{Ext}_{Q}^{1}\left(N_{I}, Q_{P}\right)$ and thus nonsingularity of $N$. 


\section{Cell decomposition}

Let $Q$ be a Dynkin quiver, $P$ and $I$ respectively a projective and an injective representation of $Q$. Let $M:=P \oplus I$ and let $\mathrm{Gr}=\mathrm{Gr}_{\boldsymbol{e}}(M)$ where $\boldsymbol{e}=\operatorname{dim} P$. In this section we construct a cellular decomposition of $\mathrm{Gr}$.

The indecomposable direct summands of $M$ are either injective or projective. In particular they are thin, that is, the vector space at every vertex is at most one-dimensional. The set of generators of these one-dimensional spaces form a linear basis of $M$ which we denote by $\boldsymbol{B}$. To each indecomposable summand $L$ of $M$ we assign an integer $d(L)$, the degree of $\mathrm{L}$, so that if $\operatorname{Hom}_{Q}\left(L, L^{\prime}\right) \neq 0$ then $d(L)<d\left(L^{\prime}\right)$ and so that all the degrees are different. In particular the degrees of the homogeneous vectors of $I$ are strictly bigger than the ones of $P$ (in case there is a projective-injective summand in both $P$ and $I$ we choose the degree of the copy in $I$ to be bigger than the degree of the copy in $P$ ). To every vector of $L$ we assign degree $d(L)$. In particular every element $v$ of $\boldsymbol{B}$ has an assigned degree $d(v)$. In view of [Cerulli Irelli 2011] the one-dimensional torus $T=\mathbb{C}^{*}$ acts on $\mathrm{Gr}$ as follows: for every $v \in \boldsymbol{B}$ and every $\lambda \in T$ we define

$$
\lambda \cdot v:=\lambda^{d(v)} v .
$$

This action extends uniquely to an action on $M$ and induces an action on Gr. The $T$-fixed points are precisely the points of Gr generated by a part of $\boldsymbol{B}$, that is, the coordinate subrepresentations of $P \oplus I$ of dimension vector $\operatorname{dim} P$.

We denote the (finite) set of $T$-fixed points of $\mathrm{Gr}$ by $\mathrm{Gr}^{T}$.

For every $L \in \mathrm{Gr}^{T}$, the torus acts on the tangent space $T_{L}(\mathrm{Gr}) \simeq \operatorname{Hom}_{Q}(L, M / L)$. More explicitly, the vector space $\operatorname{Hom}_{Q}(L, M / L)$ has a basis given by elements which associate to a basis vector $v \in L \cap \boldsymbol{B}$ a nonzero element $v^{\prime} \in M / L \cap \boldsymbol{B}$ and such element is homogeneous of degree $d\left(v^{\prime}\right)-d(v)$ [Crawley-Boevey 1989]. We denote by $\operatorname{Hom}_{Q}(L, M / L)^{+}$the vector subspace of $\operatorname{Hom}_{Q}(L, M / L)$ generated by the basis elements of positive degree.

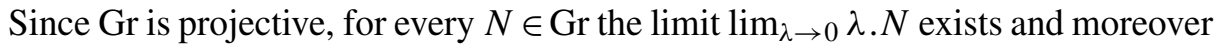
it is $T$-fixed; see for example [Chriss and Ginzburg 1997, Lemma 2.4.3]. For every $L \in \mathrm{Gr}^{T}$ we consider its attracting set

$$
\mathscr{C}(L)=\left\{N \in \mathrm{Gr} \mid \lim _{\lambda \rightarrow 0} \lambda \cdot N=L\right\} .
$$

The action (5-1) on Gr induces an action on $\mathrm{Gr}_{f}(I)$ and $\mathrm{Gr}_{\boldsymbol{e}-\boldsymbol{f}}(P)$ so that the map

$$
\zeta: \mathscr{Y}_{f} \rightarrow \operatorname{Gr}_{f}(I) \times \operatorname{Gr}_{e-f}(P)
$$

is $T$-equivariant. Since both $\mathrm{Gr}_{f}(I)$ and $\mathrm{Gr}_{e-f}(P)$ are smooth $(P$ and $I$ being rigid), we apply [Białynicki-Birula 1973] and we get cellular decompositions into 
attracting sets

$$
\operatorname{Gr}_{f}(I)=\bigsqcup_{L_{I} \in \operatorname{Gr}_{f}(I)^{T}} \mathscr{C}\left(L_{I}\right) \text { and } \operatorname{Gr}_{g}(P)=\bigsqcup_{L_{P} \in \operatorname{Gr}_{g}(P)^{T}} \mathscr{C}\left(L_{P}\right) \text {, }
$$

and moreover $\mathscr{C}\left(L_{I}\right) \simeq \operatorname{Hom}_{Q}\left(L_{I}, I / L_{I}\right)^{+}$and $\mathscr{C}\left(L_{P}\right) \simeq \operatorname{Hom}_{Q}\left(L_{P}, P / L_{P}\right)^{+}$.

Theorem 5.1. The attracting set of each $L \in \mathrm{Gr}^{T}$ is an affine space isomorphic to $\operatorname{Hom}_{Q}(L, M / L)^{+}$. In particular, we get a cellular decomposition

Moreover,

$$
\mathrm{Gr}=\coprod_{L \in \mathrm{Gr}^{T}} \mathscr{C}(L)
$$

$$
\mathscr{C}(L)=\zeta^{-1}\left(\mathscr{C}\left(L_{I}\right) \times \mathscr{C}\left(L_{P}\right)\right) \simeq \mathscr{C}\left(L_{I}\right) \times \mathscr{C}\left(L_{P}\right) \times \operatorname{Hom}_{Q}\left(L_{P}, I / L_{I}\right) .
$$

Proof. The subvariety $\mathscr{Y}_{f}:=\zeta^{-1}\left(\operatorname{Gr}_{f}(I) \times \operatorname{Gr}_{\operatorname{dim} P-f}(P)\right)$ is smooth but not projective. Nevertheless it enjoys the following property:

$$
\lim _{\lambda \rightarrow 0} \lambda \cdot N \in \mathscr{Y}_{f} \text { for all } N \in \mathscr{Y}_{\boldsymbol{f}} .
$$

Indeed let $N$ be a point of $\mathscr{Y}_{\boldsymbol{f}}$, and let $w_{1}, \ldots, w_{|\boldsymbol{e}|}$ be a basis of it (here $|\boldsymbol{e}|=$ $\left.\sum_{i \in Q_{0}} e_{i}\right)$. We write every $w_{i}$ in the basis $\boldsymbol{B}$, and we find a vector $v_{i} \in \boldsymbol{B}$ which has minimal degree in this linear combination and whose coefficient can be assumed to be 1 . We call $v_{i}$ the leading term of $w_{i}$. The subrepresentation $N_{I}=N \cap I$ is generated by those $w_{i}$ which belong to $I$ while $N_{P}=\pi(N) \simeq N / N_{I}$ is generated by the remaining ones. The torus action is chosen in such a way that the leading term of every $w_{j} \in N_{P}$ belongs to $\mathrm{P}$. The limit point $L:=\lim _{\lambda \rightarrow 0} \lambda \cdot N$ has $v_{1}, \ldots, v_{|\boldsymbol{e}|}$ as its basis. The subrepresentation $L_{I}=L \cap I$ is generated precisely by the $v_{i}$ which are the leading terms of $w_{i} \in N_{I}$. In particular $\operatorname{dim} L_{I}=\operatorname{dim} N_{I}=\boldsymbol{f}$ and hence $L \in \mathrm{Gr}_{f}$.

Since the map $\zeta$ is $T$-equivariant, (5-3) follows from (5-4).

It remains to prove that $\mathscr{C}(L) \simeq \operatorname{Hom}_{Q}(L, M / L)^{+}$. This is a consequence of

$$
\begin{aligned}
& \mathscr{C}\left(L_{I}\right) \simeq \operatorname{Hom}_{Q}\left(L_{I}, I / L_{I}\right)^{+}, \quad \operatorname{Hom}_{Q}\left(L_{I}, P / L_{I}\right)^{+}=0, \\
& \mathscr{C}\left(L_{P}\right) \simeq \operatorname{Hom}_{Q}\left(L_{P}, P / L_{P}\right)^{+}, \quad \operatorname{Hom}_{Q}\left(L_{P}, I / L_{I}\right)^{+}=\operatorname{Hom}_{Q}\left(L_{P}, I / L_{I}\right),
\end{aligned}
$$

together with the isomorphism (5-3).

The following example shows that for $L \in \mathrm{Gr}^{T}$ and $N \in \mathscr{C}(L)$, it is not true that the tangent spaces at $\mathrm{N}$ and $\mathrm{L}$ have the same dimension.

Example 5.2. Let

$$
Q:=\quad 1 \longrightarrow 2 \longrightarrow>^{3}
$$


be a Dynkin quiver of type $D_{4}$. For every vertex $k \in Q_{0}$, let $P_{k}$ and $I_{k}$ be, respectively, the indecomposable projective and injective Q-representations at vertex $k$. Let $P:=P_{1} \oplus P_{2} \oplus P_{3} \oplus P_{4}$ and $I:=I_{1} \oplus I_{2} \oplus I_{3} \oplus I_{4}$. We consider the variety $\operatorname{Gr}_{(1233)}(I \oplus P)$. We assign degree $\operatorname{deg}\left(P_{k}\right):=4-k$ and $\operatorname{deg}\left(I_{k}\right):=4+k$ for $k=1,2,3,4$. We notice that $I_{4} \oplus I_{3} \oplus I_{2}$ has an indecomposable subrepresentation $N_{I}$ of dimension vector (1211) such that $\lim _{\lambda \rightarrow 0} \lambda \cdot N_{I}=I_{4} \oplus(0110):=L$, where (0110) denotes the indecomposable subrepresentation of $I_{3}$ of dimension vector (0110). We have $I / L_{I} \simeq I / N_{I} \simeq I_{1} \oplus I_{1} \oplus I_{2}$ and $\operatorname{dim} \operatorname{Hom}_{Q}\left(N_{I}, I / N_{I}\right)=$ $\operatorname{dim} \operatorname{Hom}_{Q}\left(L_{I}, I / L_{I}\right)=3$. Let us choose $L_{P}$ inside $P$ of dimension vector (0022) so that $L_{I} \oplus L_{P} \in \mathrm{Gr}$. We choose $L_{P} \simeq P_{3}^{2} \oplus P_{4}^{2}$, where $P_{3}^{2}$ is a subrepresentation of $P_{1} \oplus P_{3}$ and $P_{4}^{2}$ is in $P_{1} \oplus P_{2}$. The quotient $P / L_{P} \simeq I_{2} \oplus(0110) \oplus P_{4}$. Now $\operatorname{dim} \operatorname{Ext}_{Q}^{1}\left(N_{I}, P / L_{P}\right)=\operatorname{dim}_{\operatorname{Ext}_{Q}}^{1}\left(N_{I}, P_{4}\right)=1$, but $\operatorname{dim} \operatorname{Ext}_{Q}^{1}\left(L_{I}, P / L_{P}\right)=$ $\operatorname{dim} \operatorname{Ext}_{Q}^{1}\left(I_{4},(0110)\right)+\operatorname{dim} \operatorname{Ext}_{Q}^{1}\left((0110), P_{4}\right)=2$.

\section{Poincaré polynomials in type $A$ and Genocchi numbers}

In this section we compute the Poincaré polynomials of $\mathrm{Gr}_{\operatorname{dim} P}(P \oplus I)$ for equioriented quiver of type $A$ and derive some combinatorial consequences.

Equioriented quiver of type A. For two nonnegative integers $k$ and $l$, the $q$-binomial coefficient $\left(\begin{array}{l}k \\ l\end{array}\right)_{q}$ is defined by the formula

$$
\left(\begin{array}{l}
k \\
l
\end{array}\right)_{q}=\frac{k_{q} !}{l_{q} !(k-l)_{q} !} \text {, where } k_{q} !=(1-q)\left(1-q^{2}\right) \ldots\left(1-q^{k}\right) .
$$

We also set $\left(\begin{array}{l}k \\ l\end{array}\right)_{q}=0$ if $k<l, k<0$, or $l<0$.

Recall (see Proposition 2.7) that $\mathcal{F}_{n+1}^{a}$ is isomorphic to $\operatorname{Gr}_{\operatorname{dim} P}(P \oplus I)$, where $P$ and $I$ are the direct sums of, respectively, all projective and all injective indecomposable representations of $Q$. According to Proposition 4.4, in order to compute the Poincaré polynomial of $\mathrm{Gr}_{\boldsymbol{e}}(P \oplus I)$, we only need to compute the Poincaré polynomials of $\mathrm{Gr}_{g}(P)$ and $\mathrm{Gr}_{f}(I)$ for arbitrary dimension vectors $\boldsymbol{g}=\left(g_{1}, \ldots, g_{n}\right)$ and $\boldsymbol{f}=\left(f_{1}, \ldots, f_{n}\right)$. Let us compute these polynomials in a slightly more general setting. Namely, fix two collections of nonnegative integers $a_{1}, \ldots, a_{n}$ and $b_{1}, \ldots, b_{n}$, and set $P=\bigoplus_{i=1}^{n} P_{i}^{a_{i}}$ and $I=\bigoplus_{i=1}^{n} I_{i}^{b_{i}}$.

Lemma 6.1. We have

$$
\begin{aligned}
& P_{G r_{g}(P)}(q)=\prod_{k=1}^{n}\left(\begin{array}{c}
a_{1}+\cdots+a_{k}-g_{k-1} \\
g_{k}-g_{k-1}
\end{array}\right)_{q}, \\
& P_{G r_{f}(I)}(q)=\prod_{k=1}^{n}\left(\begin{array}{c}
b_{n+1-k}+f_{n+2-k} \\
f_{n+1-k}
\end{array}\right)_{q}
\end{aligned}
$$

with the convention $g_{0}=0, f_{n+1}=0$. 
Proof. We first prove the first formula by induction on $n$. For $n=1$, the formula reduces to the well-known formula for the Poincare polynomials of the classical Grassmannians. Let $n>1$. Consider the map $\operatorname{Gr}_{g}(P) \rightarrow \operatorname{Gr}_{g_{1}}\left((P)_{1}\right)$. We claim that this map is a fibration with the base $\mathrm{Gr}_{g_{1}}\left(\mathbb{C}^{a_{1}}\right)$ and a fiber isomorphic to $\operatorname{Gr}_{\left(g_{2}-g_{1}, g_{3}-g_{1}, \ldots, g_{n}-g_{1}\right)}\left(P_{1}^{a_{1}-g_{1}} \bigoplus_{i=2}^{n} P_{i}^{a_{i}}\right)$. In fact, an element of $\operatorname{Gr}_{g}(P)$ is a collection of spaces $\left(V_{1}, \ldots, V_{n}\right)$ such that $V_{i} \subset(P)_{i}$. We note that all the maps in $P$ corresponding to the arrows $i \rightarrow i+1$ are embeddings. Therefore, if one fixes a $g_{1}$-dimensional subspace $V_{1} \subset P_{1}$, this automatically determines the $g_{1}$-dimensional subspaces to be contained in $V_{2}, \ldots, V_{n}$. This proves the claim. Now formula (6-1) follows by induction.

In order to prove (6-2), we consider the map

$$
\operatorname{Gr}_{\boldsymbol{f}}(I) \rightarrow \operatorname{Gr}_{f^{*}}\left(I^{*}\right): N \mapsto\left\{\varphi \in I^{*} \mid \varphi(N)=0\right\},
$$

where $I^{*}=\operatorname{Hom}_{\mathbb{C}}(I, \mathbb{C})$ and $\boldsymbol{f}^{*}=\left(f_{1}^{*}, \cdots, f_{n-1}^{*}\right)=\operatorname{dim} I-\boldsymbol{f}$ is defined by

$$
f_{k}^{*}=b_{k}+b_{k+1}+\cdots+b_{n}-f_{k} .
$$

Now $I^{*}$ can be identified with $\bigoplus_{i=1}^{n} P_{n+1-i}^{b_{i}}$ by acting on the vertices of $Q$ with the permutation $\omega: i \mapsto n-i$ for every $i=1,2, \cdots, n-1$. We hence have an isomorphism

$$
\operatorname{Gr}_{f}(I) \simeq \operatorname{Gr}_{\omega f^{*}}\left(\bigoplus_{i=1}^{n} P_{i}^{b_{i}}\right)
$$

Substituting into (6-1), we obtain (6-2).

Theorem 6.2. Let $\mathrm{Gr}=\mathrm{Gr}_{\boldsymbol{e}}(I \oplus P)$ with $I$ and $P$ as above. Then the Poincaré polynomial $P_{\mathrm{Gr}}(q)$ of $\mathrm{Gr}$ is given by

$$
\sum_{\boldsymbol{f}+\boldsymbol{g}=\boldsymbol{e}} q^{\sum_{i=1}^{n} g_{i}\left(a_{i}-f_{i}+f_{i+1}\right)} \prod_{k=1}^{n}\left(\begin{array}{c}
a_{1}+\cdots+a_{k}-g_{k-1} \\
g_{k}-g_{k-1}
\end{array}\right)_{q} \prod_{k=1}^{n}\left(\begin{array}{c}
b_{n+1-k}+f_{n+2-k} \\
f_{n+1-k}
\end{array}\right)_{q} .
$$

Proof. Recall the decomposition $\operatorname{Gr}_{e}(P \oplus I)=\sqcup_{f} \mathscr{Y}_{f}$. Each stratum $\mathscr{Y}_{f}$ is a total space of a vector bundle over $\operatorname{Gr}_{g}(P) \times \mathrm{Gr}_{f}(I)$ with fiber over a point $\left(N_{P}, N_{I}\right) \in$ $\operatorname{Gr}_{g}(P) \times \operatorname{Gr}_{f}(I)$ isomorphic to $\operatorname{Hom}_{Q}\left(N_{P}, I / N_{I}\right)$. Since $\operatorname{Ext}_{Q}^{1}\left(N_{P}, I / N_{I}\right)=0$, we obtain $\operatorname{dim} \operatorname{Hom}_{Q}\left(N_{P}, I / N_{I}\right)=\langle\boldsymbol{g}, \operatorname{dim} I-\boldsymbol{f}\rangle$. Since $Q$ is the equioriented quiver of type $A_{n}$, we obtain

$$
\langle\boldsymbol{g}, \operatorname{dim} I-\boldsymbol{f}\rangle=\sum_{i=1}^{n} g_{i}\left(a_{i}-f_{i}+f_{i+1}\right)
$$

Now our theorem follows from formulas (6-1) and (6-2).

Now let $a_{i}=b_{i}=1, i=1, \ldots, n$. Then the quiver $\operatorname{Grassmannian}_{\operatorname{Gr}_{\operatorname{dim}} P(P \oplus I)}$ is isomorphic to $\mathcal{F}_{n+1}^{a}$. 
Corollary 6.3. The Poincaré polynomial of the complete degenerate flag variety $\mathcal{F}_{n+1}^{a}$ is equal to

$$
\sum_{f_{1}, \ldots, f_{n} \geq 0} q^{\sum_{k=1}^{n}\left(k-f_{k}\right)\left(1-f_{k}+f_{k+1}\right)} \prod_{k=1}^{n}\left(\begin{array}{c}
1+f_{k-1} \\
f_{k}
\end{array}\right)_{q} \prod_{k=1}^{n}\left(\begin{array}{c}
1+f_{k+1} \\
f_{k}
\end{array}\right)_{q}
$$

where $f_{0}=f_{n+1}=0$.

Now fix a collection $\boldsymbol{d}=\left(d_{1}, \ldots, d_{s}\right)$ with $0=d_{0}<d_{1}<\cdots<d_{s}<d_{s+1}=n+1$.

Corollary 6.4. Define $a_{i}=d_{i}-d_{i-1}$ and $b_{i}=d_{i+1}-d_{i}$. Then Theorem 6.2 gives the Poincaré polynomial of the partial degenerate flag variety $\mathcal{F}_{\boldsymbol{d}}^{a}$.

Proof. This follows from Proposition 2.9.

The normalized median Genocchi numbers. Recall that the Euler characteristic of $\mathcal{F}_{n+1}^{a}$ is equal to the $(n+1)$-st normalized median Genocchi number $h_{n+1}$; see [Feigin 2011, Proposition 3.1 and Corollary 3.7]. In particular, the Poincaré polynomial (6-4) provides natural $q$-deformation $h_{n+1}(q)$. We also arrive at the following formula.

Corollary 6.5. With the convention $f_{0}=f_{n+1}=0$, we have

$$
h_{n+1}=\sum_{f_{1}, \ldots, f_{n} \geq 0} \prod_{k=1}^{n}\left(\begin{array}{c}
1+f_{k-1} \\
f_{k}
\end{array}\right) \prod_{k=1}^{n}\left(\begin{array}{c}
1+f_{k+1} \\
f_{k}
\end{array}\right)
$$

We note that formula (6-5) can be seen as a sum over the set $M_{n+1}$ of Motzkin paths starting at $(0,0)$ and ending at $(n+1,0)$. Namely, we note that a term in (6-5) is zero unless, for all $i=1, \ldots, n$, we have $f_{i+1}=f_{i}, f_{i+1}=f_{i}+1$, or $f_{i+1}=f_{i}-1$ (recall that $f_{i} \geq 0$ and $f_{0}=f_{n+1}=0$ ). Therefore the terms in (6-5) are labeled by Motzkin paths; see for example [Donaghey and Shapiro 1977]. We can simplify the expression for $h_{n+1}$. Namely, for a Motzkin path $f \in M_{n+1}$ let $l(\boldsymbol{f})$ be the number of rises $\left(f_{i+1}=f_{i}+1\right)$ plus the number of falls $\left(f_{i+1}=f_{i}-1\right)$. Then we obtain:

Corollary 6.6.

$$
h_{n+1}=\sum_{f \in M_{n+1}} \frac{\prod_{k=1}^{n}\left(1+f_{k}\right)^{2}}{2^{l(f)}} .
$$

We note also that Remark 4.2 produces one more combinatorial definition of the numbers $h_{n+1}$. Namely, for $1 \leq i \leq j \leq n$, we denote by $S_{i, j}$ the indecomposable representation of $Q$ such that

$$
\operatorname{dim} S_{i, j}=(\underbrace{0, \ldots, 0}_{i-1}, \underbrace{1, \ldots, 1}_{j-i+1}, 0, \ldots, 0) .
$$


In particular, the simple indecomposable representation $S_{i}$ coincides with $S_{i, i}$. Then we have $\operatorname{dim} \operatorname{Hom}_{Q}\left(S_{k}, S_{i, j}\right)=\left\{\begin{array}{ll}1 & \text { if } k=j, \\ 0 & \text { otherwise; }\end{array} \quad \operatorname{dim} \operatorname{Hom}_{Q}\left(S_{i, j}, S_{k}\right)= \begin{cases}1 & \text { if } i=k, \\ 0 & \text { otherwise. }\end{cases}\right.$

Recall (see Theorem 4.1) that the Euler characteristic of $\mathcal{F}_{n+1}^{a}$ is equal to the number of isomorphism classes of pairs ([ $\left.\left.Q_{P}\right],\left[N_{I}\right]\right)$ such that $N_{I}$ is embedded into $I=\bigoplus_{k=1}^{n} I_{k}, Q_{P}$ is a quotient of $P=\bigoplus_{k=1}^{n} P_{k}$, and $\operatorname{dim} N_{I}=\operatorname{dim} Q_{P}$. Let

$$
N_{I}=\bigoplus_{1 \leq i \leq j \leq n} S_{i, j}^{r_{i, j}} \quad \text { and } \quad Q_{P}=\bigoplus_{1 \leq i \leq j \leq n} S_{i, j}^{m_{i, j}} .
$$

Then from Remark 4.2 we obtain the following proposition.

Proposition 6.7. The normalized median Genocchi number $h_{n+1}$ is equal to the number of pairs of collections of nonnegative integers $\left(r_{i, j}\right),\left(m_{i, j}\right), 1 \leq i \leq j \leq n$, subject to the following conditions for all $k=1, \ldots, n$ :

$$
\sum_{k=i}^{n} r_{i, k} \leq 1, \quad \sum_{k=1}^{j} m_{k, j} \leq 1, \quad \sum_{i \leq k \leq j} r_{i, j}=\sum_{i \leq k \leq j} m_{i, j} .
$$

\section{Cells and the group action in type $A$}

In this section we fix $Q$ to be the equioriented quiver of type $A_{n}$. Description of the group. Let $P=\bigoplus_{i=1}^{n} P_{i}$ and $I=\bigoplus_{i=1}^{n} I_{i}$. As in the general case,
we consider the group

$$
G=\left[\begin{array}{cc}
\operatorname{Aut}_{Q}(P) & 0 \\
\operatorname{Hom}_{Q}(P, I) & \operatorname{Aut}_{Q}(I)
\end{array}\right],
$$

which is a subgroup of $\operatorname{Aut}_{Q}(P \oplus I)$.

Remark 7.1. The whole group of automorphisms $\operatorname{Aut}_{Q}(P \oplus I)$ is generated by $G$ and $\exp \left(\operatorname{Hom}_{Q}(I, P)\right)$. We note that $\operatorname{Hom}_{Q}(I, P)$ is a one-dimensional space. In fact, $\operatorname{Hom}_{Q}\left(I_{k}, P_{l}\right)=0$ unless $k=n, i=1$, and $I_{n} \simeq P_{1}$. Thus $G$ "almost" coincides with $\operatorname{Aut}(P \oplus I)$.

We now describe $G$ explicitly.

Lemma 7.2. The groups $\operatorname{Aut}_{Q}(P)$ and $\operatorname{Aut}_{Q}(I)$ are isomorphic to the Borel subgroup $B_{n}$ of the Lie group $G L_{n}$, that is, to the group of nondegenerate uppertriangular matrices.

Proof. For $g \in \operatorname{Aut}(P \oplus I)$, let $g_{i}$ be the component acting on $(P \oplus I)_{i}$ (the vector space corresponding to the $i$-th vertex). Then the map $g \mapsto g_{n}$ gives a group isomorphism $\operatorname{Aut}_{Q}(P) \simeq B_{n}$. In fact, if $k>l$, $\operatorname{Hom}_{Q}\left(P_{k}, P_{l}\right)=0$; otherwise, 
$\operatorname{Hom}_{Q}\left(P_{k}, P_{l}\right)$ is one-dimensional and is completely determined by the $n$-th component. Similarly, the map $g \mapsto g_{1}$ gives a group isomorphism $\operatorname{Aut}_{Q}(I) \simeq B_{n}$.

In what follows, we denote $\operatorname{Aut}_{Q}(P)$ by $B_{P}$ and $\operatorname{Aut}_{Q}(I)$ by $B_{I}$.

Proposition 7.3. The group $G$ is isomorphic to the semidirect product

$$
\mathbb{G}_{a}^{n(n+1) / 2} \rtimes\left(B_{P} \times B_{I}\right) .
$$

Proof. First, the groups $B_{P}$ and $B_{I}$ commute inside $G$. Second, the group $G$ is generated by $B_{P}, B_{I}$, and $\exp \left(\operatorname{Hom}_{Q}(P, I)\right)$. The group $\exp \left(\operatorname{Hom}_{Q}(P, I)\right)$ is abelian and isomorphic to $\mathbb{G}_{a}^{n(n+1) / 2}$ (the abelian version of the unipotent subgroup of the lower-triangular matrices in $\left.\mathrm{SL}_{n+1}\right)$. In fact, if $i>j, \operatorname{Hom}_{Q}\left(P_{i}, I_{j}\right)$ is trivial; otherwise, it is one-dimensional. Also, $\exp \left(\operatorname{Hom}_{Q}(P, I)\right)$ is normal in $G$.

We now describe explicitly the structure of the semidirect product. For this we pass to the level of the Lie algebras. So let $\mathfrak{b}_{P}$ and $\mathfrak{b}_{I}$ be the Lie algebras of $B_{P}$ and $B_{I}$, respectively $\left(\mathfrak{b}_{P}\right.$ and $\mathfrak{b}_{I}$ are isomorphic to the Borel subalgebra of $\left.\mathfrak{s l}_{n}\right)$. Let $\left(\mathfrak{n}^{-}\right)^{a}$ be the abelian $n(n+1) / 2$-dimensional Lie algebra, that is, the Lie algebra of the group $\mathbb{G}_{a}^{n(n+1) / 2}$. Also, let $\mathfrak{b}$ be the Borel subalgebra of $\mathfrak{s l}_{n+1}$. Recall that the degenerate Lie algebra $\mathfrak{s l}_{n+1}^{a}$ is defined as $\left(\mathfrak{n}^{-}\right)^{a} \oplus \mathfrak{b}$, where $\left(\mathfrak{n}^{-}\right)^{a}$ is an abelian ideal and the action of $\mathfrak{b}$ on $\left(\mathfrak{n}^{-}\right)^{a}$ is induced by the adjoint action of $\mathfrak{b}$ on the quotient $\left(\mathfrak{n}^{-}\right)^{a} \simeq \mathfrak{s l}_{n+1} / \mathfrak{b}$. Consider the embedding $\iota_{P}: \mathfrak{b}_{P} \rightarrow \mathfrak{b}, E_{i, j} \mapsto E_{i, j}$, and the embedding $\iota_{I}: \mathfrak{b}_{I} \rightarrow \mathfrak{b}, E_{i, j} \mapsto E_{i+1, j+1}$. These embeddings define the structures of $\mathfrak{b}_{P}$ - and $\mathfrak{b}_{I}$-modules on $\left(\mathfrak{n}^{-}\right)^{a}$.

Proposition 7.4. The Lie algebra of the group $G$ is isomorphic to $\left(\mathfrak{n}^{-}\right)^{a} \oplus \mathfrak{b}_{P} \oplus \mathfrak{b}_{I}$, where $\left(\mathfrak{n}^{-}\right)^{a}$ is an abelian ideal and the structure of $\mathfrak{b}_{P} \oplus \mathfrak{b}_{I}$-module on $\left(\mathfrak{n}^{-}\right)^{a}$ is defined by the embeddings $\iota_{P}$ and $\iota_{I}$.

Proof. The Lie algebra of $G$ is isomorphic to the direct $\operatorname{sum}_{\operatorname{End}_{Q}}(P) \oplus \operatorname{End}_{Q}(I) \oplus$ $\operatorname{Hom}_{Q}(P, I)$. Recall that the identification $\operatorname{Hom}_{Q}(P, P) \simeq \mathfrak{b}_{P}$ is given by $a \mapsto a_{n}$ and the identification $\operatorname{Hom}_{Q}(I, I) \simeq \mathfrak{b}_{I}$ is given by $a \mapsto a_{1}$, where $a_{i}$ denotes the $i$-th component of $a \in \operatorname{Hom}_{Q}(P \oplus I, P \oplus I)$. Recall from page 173 that $(P \oplus I)_{1}$ is spanned by the vectors $w_{1, j}, j=1, \ldots, n+1$, and that $w_{1,1} \in\left(P_{1}\right)_{1}$ and $w_{1, j} \in\left(I_{j-1}\right)_{1}$ for $j>1$. Therefore, we have a natural embedding $\mathfrak{b}_{I} \subset \mathfrak{b}$ mapping the matrix unit $E_{i, j}$ to $E_{i+1, j+1}$. Similarly, $(P \oplus I)_{n}$ is spanned by the vectors $w_{n, j}$, $j=1, \ldots, n+1$, together with $w_{n, n+1} \in\left(I_{n}\right)_{n}$ and $w_{n, j} \in\left(P_{j}\right)_{n}$ for $j<n+1$, giving the natural embedding $\mathfrak{b}_{I} \subset \mathfrak{b}, E_{i, j} \mapsto E_{i, j}$. With such a description, it is easy to compute the commutator of an element of $\mathfrak{b}_{P} \oplus \mathfrak{b}_{I}$ with an element of $\operatorname{Hom}_{Q}(P, I) \simeq\left(\mathfrak{n}^{-}\right)^{a}$.

We now compare $G$ with $\mathrm{SL}_{n+1}^{a}$. We note that the Lie algebra $\mathfrak{s l}_{n+1}^{a}$ and the Lie group $\mathrm{SL}_{n+1}^{a}$ have one-dimensional centers. Namely, let $\theta$ be the highest root of $\mathfrak{s l}_{n+1}$ and let $e_{\theta}=E_{1, n} \in \mathfrak{b} \subset \mathfrak{s l}_{n+1}$ be the corresponding element. Then $e_{\theta}$ 
commutes with everything in $\mathfrak{s l}_{n+1}^{a}$ and thus the exponents $\exp \left(t e_{\theta}\right) \in \mathrm{SL}_{n+1}^{a}$ form the center $Z$. From Proposition 7.4 we obtain the following corollary.

Corollary 7.5. The group $\mathrm{SL}_{n+1}^{a} / Z$ is embedded into $G$.

Bruhat-type decomposition. The goal of this subsection is to study the $G$-orbits on the degenerate flag varieties. So let $\boldsymbol{d}=\left(d_{1}, \ldots, d_{s}\right)$ for $0=d_{0}<d_{1}<\cdots<$ $d_{s}<d_{s+1}=n+1$.

Lemma 7.6. The group $G$ acts naturally on all degenerate flag varieties $\mathcal{F}_{\boldsymbol{d}}^{a}$.

Proof. By definition, $G$ acts on the degenerate flag variety $\mathcal{F}_{n+1}^{a}$. We note that there exists a map $\mathcal{F}_{n+1}^{a} \rightarrow \mathcal{F}_{\boldsymbol{d}}^{a}$ defined by $\left(V_{1}, \ldots, V_{n}\right) \mapsto\left(V_{d_{1}}, \ldots, V_{d_{s}}\right)$. Since $G$ acts fiberwise with respect to this projection, the $G$-action on $\mathcal{F}_{n+1}^{a}$ induces a $G$-action on $\mathcal{F}_{\boldsymbol{d}}^{a}$.

We first work out the case $s=1$, that is, the $G$-action on the classical Grassmannian $\mathrm{Gr}_{d}(n+1)$. We first recall the cellular decomposition from [Feigin 2011]. The cells are labeled by torus fixed points, that is, by collections $L=\left(l_{1}, \ldots, l_{d}\right)$ with $1 \leq l_{1}<\cdots<l_{d} \leq n+1$. The corresponding cell is denoted by $\mathscr{C}_{L}$. Explicitly, the elements of $\mathscr{C}_{L}$ can be described as follows. Let $k$ be an integer such that $l_{k} \leq d<l_{k+1}$. Recall the basis $w_{1}, \ldots, w_{n+1}$ of $W=\mathbb{C}^{n+1}$. We denote by $x_{L} \in \operatorname{Gr}_{d+1}(n+1)$ the linear span of $w_{l_{1}}, \ldots, w_{l_{d}}$. Then a $d$-dimensional subspace $V$ belongs to $\mathscr{C}_{L}$ if and only if it has a basis $e_{1}, \ldots, e_{d}$ such that for some constants $c_{p}$, we have

$$
\begin{gathered}
e_{j}=w_{l_{j}}+\sum_{p=1}^{l_{j}-1} c_{p} w_{p}+\sum_{p=d+1}^{n+1} c_{p} w_{p} \quad \text { for } j=1, \ldots, k ; \\
e_{j}=w_{l_{j}}+\sum_{p=d+1}^{l_{j}-1} c_{p} w_{p} \quad \text { for } j=k+1, \ldots, d .
\end{gathered}
$$

For example, $x_{L} \in \mathscr{C}_{L}$.

Lemma 7.7. Each $G$-orbit on the Grassmannian $\mathcal{F}_{(d)}^{a}$ contains exactly one torus fixed point $x_{L}$. The orbit $G \cdot x_{L}$ coincides with $\mathscr{C}_{L}$.

Proof. Follows from the definition of $G$.

We prove now that the $G$-orbits in $\operatorname{Gr}_{\operatorname{dim} P}(P \oplus I)$ described in Theorem 4.1 are cells. Moreover, we prove that this cellular decomposition coincides with the one of [Feigin 2011]. Let

$$
P=\bigoplus_{i=1}^{s} P_{i}^{d_{i}-d_{i-1}} \quad \text { and } \quad I=\bigoplus_{i=1}^{s} I_{i}^{d_{i+1}-d_{i}} .
$$

We start with the following lemma. 
Lemma 7.8. Let $N_{I} \subset I$ be a subrepresentation of I. Then there exists a unique torus fixed point $N_{I}^{\circ} \in \mathrm{Gr}_{\operatorname{dim} N_{I}}(I)$ such that $N_{I} \simeq N_{I}^{\circ}$. Similarly, for $N_{P} \subset P$ there exists a unique torus fixed point $N_{P}^{\circ} \in \mathrm{Gr}_{\operatorname{dim} N_{P}}(P)$ such that $P / N_{P} \simeq P / N_{P}^{\circ}$.

Proof. We prove the first part; the second part can be proved similarly. Recall the vectors $w_{i, j} \in\left(I_{j-1}\right)_{i}, i=1, \ldots, n, j=i+1, \ldots, n+1$ such that $w_{i, j} \mapsto w_{i+1, j}$ if $j \neq i+1$ and $w_{i, j} \mapsto 0$ if $j=i+1$. For each indecomposable summand $S_{k, l}$ of $N_{I}$ we construct the corresponding indecomposable summand of $N_{I}^{\circ}$. Namely, we take the subrepresentation in $I_{l}$ of dimension vector

$$
(\underbrace{0, \ldots, 0}_{k-1}, \underbrace{1, \ldots, 1}_{l-k+1}, 0, \ldots, 0) .
$$

Since each $I_{l}$ is torus-fixed, our lemma is proved.

Remark 7.9. This lemma is not true for injective modules over Dynkin quivers in general. Namely, consider the quiver from Example 4.3 and let $N_{I} \subset I_{3} \oplus I_{4}$ be an indecomposable $Q$-module of dimension $(1,2,1,1)$. Then, for such $N_{I}$, Lemma 7.8 does not hold.

Corollary 7.10. Each G-orbit in $\mathrm{Gr}_{\operatorname{dim} P}(P \oplus I)$ contains exactly one torus fixed point, and each such point is contained in some orbit.

Proof. Follows from Theorem 4.1 and Lemma 7.8.

We note that any torus fixed point in $\mathcal{F}_{\boldsymbol{d}}^{a}$ is the product of fixed points in the Grassmannians $\mathcal{F}_{\left(d_{i}\right)}^{a}, i=1, \ldots, s$. Therefore, any such point is of the form $\prod_{i=1}^{s} x_{L^{i}}$. We denote this point by $x_{L^{1}, \ldots, L^{s}}$.

Theorem 7.11. The orbit $G \cdot x_{L^{1}, \ldots, L^{s}}$ is the intersection of the quiver Grassmannian $\operatorname{Gr}_{\operatorname{dim} P}(P \oplus I)$ with the product of cells $\mathscr{C}_{L^{i}}$.

Proof. First, obviously $G \cdot x_{L^{1}, \ldots, L^{s}} \subset \mathcal{F}_{\boldsymbol{d}}^{a} \cap \prod_{i=1}^{s} \mathscr{C}_{L^{i}}$. Second, since each orbit contains exactly one torus fixed point and the intersection on the right hand side does not contain fixed points other than $x_{L^{1}, \ldots, L^{s}}$, the theorem is proved.

Corollary 7.12. The G-orbits on $\mathcal{F}_{\boldsymbol{d}}^{a}$ produce the same cellular decomposition as the one constructed in [Feigin 2011].

Proof. The cells from [Feigin 2011] are labeled by collections $L^{1}, \ldots, L^{s}$ (whenever $\left.x_{L^{1}, \ldots, L^{s}} \in \mathcal{F}_{\boldsymbol{d}}^{a}\right)$ and the corresponding cell $\mathscr{C}_{L^{1}, \ldots, L^{s}}$ is given by

$$
\mathscr{C}_{L^{1}, \ldots, L^{s}}=\mathcal{F}_{\boldsymbol{d}}^{a} \cap \prod_{i=1}^{s} \mathscr{C}_{L^{i}} .
$$


Cells and one-dimensional torus. In this subsection we show that the cellular decomposition described above coincides with the one constructed in Section 5. We describe the case of the complete flag varieties (in the parabolic case everything works in the same manner). Recall that the action of our torus is given by the formulas

$$
\lambda \cdot w_{i, j}= \begin{cases}\lambda^{2 n-j+1} w_{i, j} & \text { if } j>i, \\ \lambda^{n-j} w_{i, j} & \text { if } j \leq i .\end{cases}
$$

For $n=4$ we have the following picture (compare with (2-2)):

$$
\begin{aligned}
& \begin{array}{lr}
1 & w_{4,4} \\
\lambda & w_{3,3} \rightarrow w_{4,3}
\end{array} \\
& \lambda^{2} \quad w_{2,2} \rightarrow w_{3,2} \rightarrow w_{4,2} \\
& \lambda^{3} \quad w_{1,1} \rightarrow w_{2,1} \rightarrow w_{3,1}>w_{4,1} \\
& \lambda^{4} \quad w_{1,5} \rightarrow w_{2,5} \rightarrow w_{3,5} \rightarrow w_{4,5} \\
& \lambda^{5} \quad w_{1,4} \rightarrow w_{2,4} \rightarrow w_{3,4} \\
& \lambda^{6} \quad w_{1,3} \rightarrow w_{2,3} \\
& \lambda^{7} \quad w_{1,2}
\end{aligned}
$$

Proposition 7.13. Given a fixed point $x$ of the one-dimensional torus (7-3), the attracting $(\lambda \rightarrow 0)$-cell of $x$ coincides with the $G$-orbit $G \cdot x$.

Proof. First, consider the action of our torus on each Grassmannian $\operatorname{Gr}_{d}\left((P \oplus I)_{d}\right)$. Then formulas (7-1) and (7-2) imply that the attracting cells $(\lambda \rightarrow 0)$ coincide with the cells $\mathscr{C}_{L}$. Now Theorem 7.11 implies our proposition.

We note that the one-dimensional torus (7-3) does not belong to $\mathrm{SL}_{n+1}^{a}$ (more precisely, to the image of $\mathrm{SL}_{n+1}^{a}$ in the group of automorphisms of the degenerate flag variety). However, it does belong to a one-dimensional extension

$$
\mathrm{SL}_{n+1}^{a} \rtimes \mathbb{C}_{P B W}^{*}
$$

of the degenerate group; see [Feigin 2011, Remark 1.1]. Recall that the extended group is the Lie group of the extended Lie algebra $\mathfrak{s l}_{n+1}^{a} \oplus \mathbb{C} d_{P B W}$, where $d_{P B W}$ commutes with the generators $E_{i, j} \in \mathfrak{s l}_{n+1}$ as follows:

$$
\left[d_{P B W}, E_{i, j}\right]= \begin{cases}0 & \text { if } i<j, \\ E_{i, j} & \text { if } i>j .\end{cases}
$$

In particular, the action of the torus $\mathbb{C}_{P B W}^{*}=\left\{\exp \left(\lambda d_{P B W}\right), \lambda \in \mathbb{C}\right\}$ on $w_{i, j}$ is given by the formulas: $\lambda \cdot w_{i, j}=w_{i, j}$ if $i \geq j$ and $\lambda \cdot w_{i, j}=\lambda w_{i, j}$ if $i<j$. For example, 
for $n=4$ one has the following picture (vectors come equipped with the weights):

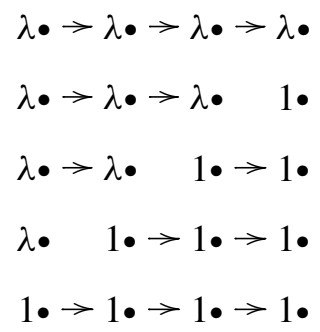

Proposition 7.14. The one-dimensional torus (7-3) sits inside the extended group $\mathrm{SL}_{n+1}^{a} \rtimes \mathbb{C}_{P B W}^{*}$.

Proof. For any collection of integers $k_{1}, \ldots, k_{n+1}$ there exists a one-dimensional torus $\mathbb{C}_{\left(k_{1}, \ldots, k_{n+1}\right)}^{*}$ inside the Cartan subgroup of $\mathrm{SL}_{n+1}^{a}$ which acts on $w_{i, j}$ by the formula $w_{i, j} \mapsto \lambda^{k_{j}} w_{i, j}$. A direct check shows that the torus (7-3) acts as

$$
\mathbb{C}_{(n, n+1, \ldots, 2 n)}^{*} \times\left(\mathbb{C}_{P B W}^{*}\right)^{-n-1} .
$$

\section{Acknowledgments}

This work was initiated during the authors' stay at the Hausdorff Research Institute for Mathematics during the trimester program "On the Interaction of Representation Theory with Geometry and Combinatorics". The hospitality and perfect working conditions of the Institute are gratefully acknowledged. Markus Reineke would like to thank K. Bongartz for helpful discussions. Giovanni Cerulli Irelli thanks C. De Concini and M. Lanini for several useful conversations at HIM and F. Esposito for enlightening suggestions.

\section{References}

[Berenstein and Zelevinsky 2005] A. Berenstein and A. Zelevinsky, "Quantum cluster algebras", Adv. Math. 195:2 (2005), 405-455. MR 2006a:20092 Zbl 1124.20028

[Białynicki-Birula 1973] A. Białynicki-Birula, "Some theorems on actions of algebraic groups", Ann. of Math. (2) 98 (1973), 480-497. MR 51 \#3186 Zbl 0275.14007

[Bongartz 1996] K. Bongartz, "On degenerations and extensions of finite-dimensional modules", $A d v$. Math. 121:2 (1996), 245-287. MR 98e:16012 Zbl 0862.16007

[Caldero and Chapoton 2006] P. Caldero and F. Chapoton, "Cluster algebras as Hall algebras of quiver representations", Comment. Mathematici Helvetici 81:3 (2006), 595-616. MR 2008b:16015 Zbl 1119.16013

[Caldero and Reineke 2008] P. Caldero and M. Reineke, "On the quiver Grassmannian in the acyclic case”, J. Pure Appl. Algebra 212:11 (2008), 2369-2380. MR 2009f:14102 Zbl 1153.14032

[Cerulli Irelli 2011] G. Cerulli Irelli, "Quiver Grassmannians associated with string modules”, J. Algebraic Combin. 33:2 (2011), 259-276. MR 2012c:16043 Zbl 05852822 
[Cerulli Irelli and Esposito 2010] G. Cerulli Irelli and F. Esposito, "Grassmannians of Kronecker type and applications to cluster algebras", preprint, 2010. To appear in Algebra and Number Theory.

[Cerulli Irelli et al. 2010] G. Cerulli Irelli, F. Esposito, and G. Dupont, "A homological interpretation of the transverse quiver Grassmannians", preprint, 2010. arXiv 1005.1405

[Chriss and Ginzburg 1997] N. Chriss and V. Ginzburg, Representation theory and complex geometry, Birkhäuser, Boston, MA, 1997. MR 98i:22021 Zbl 0879.22001

[Crawley-Boevey 1989] W. W. Crawley-Boevey, "Maps between representations of zero-relation algebras”, J. Algebra 126:2 (1989), 259-263. MR 90k:16035 Zbl 0685.16018

[Dellac 1900] H. Dellac, "Problem 1735”, L'Intermédiaire des Mathématiciens 7 (1900), 9-10.

[Donaghey and Shapiro 1977] R. Donaghey and L. W. Shapiro, "Motzkin numbers", J. Combinatorial Theory Ser. A 23:3 (1977), 291-301. MR 58 \#21644 Zbl 0417.05007

[Dumont 1974] D. Dumont, "Interprétations combinatoires des nombres de Genocchi”, Duke Math. J. 41 (1974), 305-318. MR 49 \#2412 Zbl 0297.05004

[Dumont and Randrianarivony 1994] D. Dumont and A. Randrianarivony, "Dérangements et nombres de Genocchi”, Discrete Math. 132:1-3 (1994), 37-49. MR 95h:05015 Zbl 0807.05001

[Dumont and Zeng 1994] D. Dumont and J. Zeng, "Further results on the Euler and Genocchi numbers", Aequationes Math. 47:1 (1994), 31-42. MR 95b:11021 Zbl 0805.11024

[Feigin 2010] E. Feigin, “ $\mathbb{G}_{a}^{M}$ degeneration of flag varieties”, preprint, 2010. arXiv 1007.0646

[Feigin 2011] E. Feigin, "Degenerate flag varieties and the median Genocchi numbers", preprint, 2011. arXiv 1101.1898

[Feigin and Finkelberg 2011] E. Feigin and M. Finkelberg, "Degenerate flag varieties of type A: Frobenius splitting and BWB theorem”, preprint, 2011. arXiv 1103.1491

[Fomin and Zelevinsky 2002] S. Fomin and A. Zelevinsky, "Cluster algebras. I. Foundations", J. Amer. Math. Soc. 15:2 (2002), 497-529. MR 2003f:16050

[Hartshorne 1977] R. Hartshorne, Algebraic geometry, Graduate Texts in Mathematics 52, Springer, New York, 1977. MR 57 \#3116 Zbl 0367.14001

[Matsumura 1989] H. Matsumura, Commutative ring theory, 2nd ed., Cambridge Studies in Advanced Mathematics 8, Cambridge University Press, 1989. MR 90i:13001 Zbl 0666.13002

[Qin 2010] F. Qin, "Quantum cluster variables via Serre polynomials”, preprint, 2010. arXiv 1004. 4171

[Reineke 2001] M. Reineke, "Generic extensions and multiplicative bases of quantum groups at $q=0$ ", Represent. Theory 5 (2001), 147-163. MR 2002c:17029 Zbl 1050.17015

[Reineke 2008] M. Reineke, "Framed quiver moduli, cohomology, and quantum groups", J. Algebra 320:1 (2008), 94-115. MR 2009d:16021 Zbl 1153.14033

[Schofield 1992] A. Schofield, "General representations of quivers", Proc. London Math. Soc. (3) 65:1 (1992), 46-64. MR 93d:16014 Zbl 0795.16008

[Viennot 1982] G. Viennot, "Interprétations combinatoires des nombres d'Euler et de Genocchi", Seminar on Number Theory 1981/1982:11 (1982), 94.

Communicated by Andrei Zelevinsky

Received 2011-06-16 Revised 2011-07-18 
cerulli.math@googlemail.com

evgfeig@gmail.com

reineke@math.uni-wuppertal.de
Sapienza - Università di Roma, Piazzale Aldo Moro 5, 00185 Rome, Italy

Department of Mathematics,

National Research University Higher School of Economics, Vavilova str. 7, Moscow, 117312, Russia

Fachbereich C - Mathematik, Bergische Universität Wuppertal, D-, 42097 Wuppertal, Germany 


\section{Algebra \& Number Theory}

msp.berkeley.edu/ant

\section{EDITORS}

MANAGING EDITOR

Bjorn Poonen

Massachusetts Institute of Technology

Cambridge, USA

\author{
EDITORIAL BOARD CHAIR \\ David Eisenbud \\ University of California \\ Berkeley, USA
}

\section{BOARD OF EDITORS}

Georgia Benkart

Dave Benson

Richard E. Borcherds

John H. Coates

J-L. Colliot-Thélène

Brian D. Conrad

Hélène Esnault

Hubert Flenner

Edward Frenkel

Andrew Granville

Joseph Gubeladze

Ehud Hrushovski

Craig Huneke

Mikhail Kapranov

Yujiro Kawamata

János Kollár

Yuri Manin

Barry Mazur

Philippe Michel

Susan Montgomery
University of Wisconsin, Madison, USA

University of Aberdeen, Scotland

University of California, Berkeley, USA

University of Cambridge, UK

CNRS, Université Paris-Sud, France

University of Michigan, USA

Universität Duisburg-Essen, Germany

Ruhr-Universität, Germany

University of California, Berkeley, USA

Université de Montréal, Canada

San Francisco State University, USA

Hebrew University, Israel

University of Kansas, USA

Yale University, USA

University of Tokyo, Japan

Princeton University, USA

Northwestern University, USA

Harvard University, USA

École Polytechnique Fédérale de Lausanne

University of Southern California, USA
Shigefumi Mori

Raman Parimala

Jonathan Pila

Victor Reiner

Karl Rubin

Peter Sarnak

Joseph H. Silverman

Michael Singer

Ronald Solomon

Vasudevan Srinivas

J. Toby Stafford

Bernd Sturmfels

Richard Taylor

Ravi Vakil

Michel van den Bergh

Marie-France Vignéras

Kei-Ichi Watanabe

Andrei Zelevinsky

Efim Zelmanov
RIMS, Kyoto University, Japan

Emory University, USA

University of Oxford, UK

University of Minnesota, USA

University of California, Irvine, USA

Princeton University, USA

Brown University, USA

North Carolina State University, USA

Ohio State University, USA

Tata Inst. of Fund. Research, India

University of Michigan, USA

University of California, Berkeley, USA

Harvard University, USA

Stanford University, USA

Hasselt University, Belgium

Université Paris VII, France

Nihon University, Japan

Northeastern University, USA

University of California, San Diego, USA

\section{PRODUCTION}

contact@msp.org

Silvio Levy, Scientific Editor

See inside back cover or www.jant.org for submission instructions.

The subscription price for 2012 is US \$175/year for the electronic version, and \$275/year (+\$40 shipping outside the US) for print and electronic. Subscriptions, requests for back issues from the last three years and changes of subscribers address should be sent to Mathematical Sciences Publishers, Department of Mathematics, University of California, Berkeley, CA 94720-3840, USA.

Algebra \& Number Theory (ISSN 1937-0652) at Mathematical Sciences Publishers, Department of Mathematics, University of California, Berkeley, CA 94720-3840 is published continuously online. Periodical rate postage paid at Berkeley, CA 94704, and additional mailing offices.

ANT peer review and production are managed by EditFLOW ${ }^{\circledR}$ from Mathematical Sciences Publishers.

PUBLISHED BY

mathematical sciences publishers

http://msp.org/

A NON-PROFIT CORPORATION

Typeset in IAT $_{\mathrm{E}} \mathrm{X}$

Copyright (C2012 by Mathematical Sciences Publishers 


\section{Algebra \& Number Theory}

\section{Volume $6 \quad$ No. $1 \quad 2012$}

The Chevalley-Shephard-Todd theorem for finite linearly reductive group schemes MATTHEW SATRIANO

The minimal resolution conjecture for points on del Pezzo surfaces

ROSA M. MIRÓ-ROIG and JOAN PONS-LLOPIS

$L$-series of Artin stacks over finite fields

SHENGHAO SUN

Multiplicative mimicry and improvements to the Pólya-Vinogradov inequality LEO GOLDMAKHER

Quiver Grassmannians and degenerate flag varieties

Giovanni Cerulli Irelli, Evgeny Feigin and Markus Reineke 